Cipango $\begin{aligned} & \text { Cipango } \\ & \text { Cahiers d'études japonaises }\end{aligned}$

$18 \mid 2011$

Le Japon et le fait colonial I

\title{
Les débuts de l'emprise économique japonaise en Corée coloniale, 1900-1919
}

\section{Alexandre Roy}

\section{(2) OpenEdition}

1 Journals

\section{Édition électronique}

URL : https://journals.openedition.org/cipango/1524

DOI : $10.4000 /$ cipango. 1524

ISSN : 2260-7706

Éditeur

INALCO

\section{Édition imprimée}

Date de publication : 1 juin 2011

Pagination : 135-187

ISBN : 978-2-85831-195-8

ISSN : $1164-5857$

\section{Référence électronique}

Alexandre Roy, "Les débuts de l'emprise économique japonaise en Corée coloniale, 1900-1919 », Cipango [En ligne], 18 | 2011, mis en ligne le 17 juin 2013, consulté le 30 juin 2021. URL : http:// journals.openedition.org/cipango/1524; DOl : https://doi.org/10.4000/cipango.1524

Ce document a été généré automatiquement le 30 juin 2021.

\section{(c) (1) 8}

Cipango est mis à disposition selon les termes de la Licence Creative Commons Attribution - Pas d'Utilisation Commerciale 4.0 International. 


\title{
Les débuts de l'emprise économique japonaise en Corée coloniale, 1900-1919
}

\author{
Alexandre Roy
}

1 À l'aube du xx siècle, à l'image de Kōtoku Shūsui 幸徳秋水 (1871-1911) ${ }^{1}$, peu nombreux étaient les observateurs à croire que le capitalisme japonais eût la capacité de maintenir un empire colonial sur la durée. Certes, le Japon venait de vaincre la Chine (guerre sino-japonaise, 1894-1895) et avait obtenu l'île de Taiwan ${ }^{2}$, mais le camouflet de la rétrocession immédiate du Liaodong 遼東 ${ }^{3}$ imposée par la «triple intervention » (sankoku kanshō 三国干涉, menée par la Russie, l'Allemagne et la France), la difficulté des premières années de "pacification » de l'île ainsi que la pénétration occidentale en Chine à ce même moment relativisaient grandement la présence japonaise. La victoire contre l'armée tsariste (1904-05) permit au Japon de changer la donne pour s'affirmer : récupérer ses droits sur le Liaodong (territoire à bail du " Kwantung » 関東), obtenir la moitié sud de Karafuto et ériger en Corée un protectorat doté d'un résident général (tōkan 統監) entre 1905 et 1910, puis procéder à l'« annexion » (heigō 併合) du territoire en 1910 (régime du gouvernement général, sōtoku-fu 総督府) ${ }^{4}$. En dix ans, le Japon avait constitué un empire colonial périphérique en Asie orientale, au nord de la présence coloniale française ou britannique. Si l'empire colonial sera achevé après la Première Guerre mondiale, la Corée en était alors, au début $\mathrm{du} \mathrm{xx}^{\mathrm{e}}$ siècle, la pièce centrale : colonie la plus étendue avec ses $220000 \mathrm{~km}^{2}$ ( $81 \%$ du territoire colonial) et la plus peuplée avec environ quatorze à quinze millions d'habitants en $1910^{5}$ (près d'un tiers de la population métropolitaine et plus des trois quarts de la population coloniale).

2 Le présent article se propose de réfléchir sur l'économie de la Corée coloniale durant une première période allant du début $\mathrm{du} \mathrm{xx}^{\mathrm{e}}$ siècle jusqu'au lendemain de la Première Guerre mondiale et de l'explosion de la «bulle de la guerre». Les grands panoramas historiques décrivant l'économie coloniale dans la péninsule tendent souvent à dresser des « bilans » de l'ensemble de la période coloniale, de 1905 à 1945, ou bien ne portentils que sur la période d'industrialisation massive qui caractérisa la fin de la période 
coloniale. Pourtant, la période précédant la Première Guerre mondiale nous semble relever d'une dynamique différente. Parce que la colonisation en est à ses débuts et se cherche encore, parce que la puissance économique japonaise n'est elle-même pas encore assurée en métropole et parce que, enfin, l'expansion économique japonaise de cette période est inséparable de la «bulle » de 1914-1918.

3 Nous nous proposons de nous appuyer ici sur des documents particuliers, qui sont les synthèses statistiques fournies par le gouvernement général de Corée lui-même. Ces documents ne constituent pas des sources, mais sont donc plutôt une mise en forme des documents primaires qui présentent l'activité économique dans la péninsule. Les activités japonaise et coréenne s'y côtoient, y collaborent parfois. Le regard du gouvernement général souligne l'effort fait par le Japon et met en avant les résultats obtenus. Ces données officielles méritent évidemment qu'on s'y arrête pour les décrire et les analyser.

$4 \mathrm{Au}$ moment de la constitution de son empire colonial le Japon ne pouvait soutenir à la fois son expansion extérieure et son développement économique. Mais le commencement de la guerre en Europe (1914-18) changea la donne. Les puissances occidentales concentrant leurs forces économiques en Europe, les marchés orientaux et américains s'ouvrirent largement aux produits japonais, générant rapidement un large excédent commercial (les exportations dépassant de $35 \%$ les importations) ${ }^{6}$. Les difficultés précédentes furent comme balayées, oubliées, c'était le « boum de la Grande Guerre» (taisen būmu 大戦ブーム), qui constitua la deuxième phase d'accélération du développement économique japonais après le «boum entrepreneurial » (kigyō bokkō 企 業勃興) de 1885-1890. La suspension de l'étalon-or (instauré en octobre 1897) et la fièvre aux investissements pour produire les biens devenus difficilement importables s'ajoutant à cette donne, une bulle gigantesque en résulta, renchérissant considérablement le coût de la vie. Le problème éclata à la fin de la guerre dans les « émeutes du riz » (kome sōdō 米騒動) de l'été 1918. Le gouvernement élu en septembre de cette année orchestra une politique d'accompagnement de la bulle, poussant, sur fond de relance aux États-Unis, à un regain de fièvre spéculatrice en 1919. Ce fut de courte durée : le jeu de dominos commença à s'écrouler durablement le 15 mars 1920, à l'approche de l'annonce des résultats annuels à la bourse de Tōkyō, les crédits impayés entraînèrent de nombreuses faillites bancaires et industrielles. Une crise majeure éclata, une sauvage sélection par le capital s'opéra, chamboulant la donne pour l'économie japonaise en général et son économie coloniale en particulier.

L'influence de cette crise sur l'économie coloniale en Corée impose donc la périodisation ici choisie.

6 Par ailleurs, l'historiographie laisse voir que les études de l'économie coloniale japonaise ont surtout adopté une grille de lecture englobant l'ensemble des possessions coloniales, chronologiquement (jusqu'en 1945) ou spatialement (avec Taiwan et la Mandchourie) $)^{7}$. Cette méthode a été favorisée par un débat centré sur l'opposition entre visions "positive " et "négative " du problème. La vision "positive ${ }^{8}$ tend à affirmer que l'économie coloniale a été bénéfique aux colonisés, à ignorer, atténuer ou embellir les aspects néfastes de celle-ci, ou bien à suggérer plus généralement une relation positive entre elle et la croissance économique postérieure (à Taiwan et en Corée du Sud). Ces travaux prennent souvent le soin de dire brièvement en préambule qu'il ne s'agit pas de dresser d'éloges à la colonisation, quitte à se contredire dans leur contenu'. À l'opposé, la vision « négative » est celle considérant l'économie coloniale en 
fonction de l'exploitation et de l'aliénation des colonisés, avec une tendance à focaliser sur les années 1930 et 1940. Cela regroupe les travaux du courant historiographique marxiste (d'abord japonais, comme ceux d'Asada Kyōji 浅田喬二 ${ }^{10}$ et Kaneko Fumio 金 子文夫 $\left.{ }^{11}\right)^{12}$ et nationalistes sud-coréens (dans le sens où leur objectif est de dépeindre une économie nationale meurtrie et résistante) ${ }^{13}$. Si l'opposition radicale est une caractéristique générale des études coloniales, au Japon comme ailleurs, la croissance économique en Corée du Sud et à Taiwan à partir des années 1980 a favorisé le développement du paradigme de "l'industrialisation coloniale », offrant comme un terrain d'entente ${ }^{14}$. Depuis, certains auteurs sud-coréens considèrent même l'ère de l'opposition binaire révolue ${ }^{15}$, mais un tel changement est-il possible sans changer de méthode $^{16}$ ? S'interroger sur «l'industrialisation coloniale» ne revient-il pas à considérer les premières années de la colonisation d'abord en fonction des années 1930 et $1940^{17}$ ? D'où, à nouveau, l'importance pour nous de saisir l'histoire économique des premières années de la colonisation, en accordant toute son importance au contexte particulier allant de la fin de la Guerre russo-japonaise à la fin de la Première Guerre mondiale.

7 Nous étudierons en trois temps l'évolution des infrastructures, de la production et du commerce en Corée depuis les premiers aménagements japonais sur place en 1900 jusqu'à l'avènement du Mouvement du Premier Mars $1919^{18}$.

\section{Les infrastructures : le rail et la banque}

\section{Le chemin de fer colonial}

8 En Corée, un premier pas dans l'aménagement d'infrastructures modernes fut réalisé en 1900 avec l'achèvement d'une première ligne ferroviaire par le pouvoir japonais. Jusqu'en 1910, dans le contexte des tensions géopolitiques autour de la Corée, cet effort japonais fut poursuivi afin de doter la péninsule d'un réseau de transport rapide de troupes. Dans les années 1910, l'aménagement ralentit et, économiquement, le développement de ce réseau au tracé surtout militaire rencontra quelques difficultés. La première ligne fut celle reliant la capitale, Séoul (Hansŏng), à son port, Chemulp'o (aussi appelé Inch'ŏn) : trente kilomètres de rails décidés en juin 1894 alors que le début des hostilités avec les troupes chinoises en Corée était imminent. Cette ligne était stratégique, permettant à l'armée japonaise d'accéder à la capitale coréenne rapidement après avoir été débarquée à Chemulpo (la prise de position autour de la capitale étant alors un élément clé dans le jeu de pressions militaro-diplomatiques). Si le gouvernement coréen put déjouer dans un premier temps les pressions japonaises pour accorder la concession à un entrepreneur américain (en 1896), ce dernier, connaissant des difficultés, la revendit au Japon en 1898 (démarche facilitée par l'accord passé cette année entre Tōkyō et Saint-Pétersbourg au sujet la péninsule). Il fallut toutefois au gouvernement japonais beaucoup d'efforts pour rassembler les fonds (la concession fut achetée par le gouvernement japonais 1,8 million de yens) et les entrepreneurs nécessaires (en tête desquels le financier Shibusawa Eiichi 淽沢栄一) ${ }^{19}$ et leur faire achever les travaux en juillet $1900^{20}$. Ces difficultés pour aménager cette courte ligne entre Séoul et Inch'ŏn auguraient mal de la construction de la ligne SéoulPusan 釜山, quinze fois plus longue et d'une importance stratégique majeure. 
9 Pusan était le port frontière avec le Japon, situé à deux cents kilomètres des grands ports japonais de l'ouest (Moji, Shimonoseki, Nagasaki et Hakata) : pourvu d'un chemin de fer pénétrant la péninsule, c'était la tête de pont idéale pour tout débarquement de troupes et de matériel. Comme pour la première ligne, les conditions géopolitiques auront poussé l'État japonais à prendre sur lui, d'abord pour obtenir la concession (juillet 1898) puis, dans la mesure où "d'indécisions en mesures dilatoires ${ }^{21}$ rien n'était encore fait en 1900, pour fonder en juin 1901 une compagnie responsable (Société par actions du Chemin de fer Séoul-Pusan, keipu-tetsudō kabushiki-gaisha 京釜鉄 道株式会社). Enfin, constatant la lenteur des travaux, il dut diriger directement la réalisation de la ligne à partir de la fin $1903^{22}$. La compagnie fut dotée d'un capital de vingt-cinq millions de yens à $80 \%$ empruntés en Europe, étant donné le peu de capital mobilisable au Japon. Au niveau intérieur, il fallut mener une campagne pour persuader les investisseurs japonais de fournir les quelque cinq millions de yens restants (malgré les confortables garanties de l'État et un premier versement limité à un dixième seulement de la somme engagée) ${ }^{23}$. Malgré tout, la crise financière s'abattant sur le Japon à ce moment créa de nouvelles difficultés pour obtenir les premiers versements : à l'automne 1903, seuls cent quinze kilomètres de voies ferrées sur près de quatre cents avaient été posés, et ce sur sa partie la plus plate. Inquiet, le gouvernement dut encore se charger du dossier : en novembre la compagnie fusionna avec celle de la ligne Séoul-Inch'ŏn, en décembre la nouvelle compagnie recevait d'importantes subventions et des hauts fonctionnaires furent nommés à sa tête. Trois mois plus tard, la marine japonaise déclenchait les hostilités contre la Russie et la ligne ouvrit sur toute sa longueur pour la fin des combats un an après (janvier 1905) ${ }^{24}$.

Les cinq cents kilomètres de la section nord du transcoréen, de Séoul à Sin-ŭiju (à la frontière avec la Chine) via P'yŏngyang furent eux aussi d'abord l'objet d'une concession française, mais là encore des difficultés financières firent capoter l'affaire ${ }^{25}$. La concession fut brièvement cédée à un groupe de Coréens (1899) avant d'être aussitôt reprise par le gouvernement à Séoul (1900) et sa réalisation confiée, encore, à un Français. Malgré l'inauguration des travaux en octobre 1901, l'affaire eut du mal à avancer, prise entre les pressions russes et japonaises (les camps étant divisés en leur sein quant à la politique adoptée). L'avènement de la guerre au début de 1904 permit aux Japonais d'arracher la concession ferroviaire à fins militaires : la ligne fut achevée en 1906 et son trafic marchandises et voyageurs ouvert en $1908^{26}$. Entre 1909 et 1910 , une petite ligne de cinquante-cinq kilomètres reliant P'yŏngyang au port de Chinnamp'o compléta un ensemble alors long de $1221 \mathrm{~km}$.

11 Avec la nationalisation des chemins de fer au Japon en 1906, les lignes de Corée furent rachetées et placées sous le contrôle direct de l'armée ${ }^{27}$. L'appréhension d'une guerre « revancharde » de la part de la Russie ${ }^{28}$ poussa à relier les rails sud-mandchourien et coréens pour créer un réseau continental japonais allant de Pusan à Changchun. Tōkyō obtint ainsi du gouvernement chinois en 1910 l'autorisation de transformer la simple ligne militaire établie pendant la guerre contre la Russie entre le port d'Andong (actuel Dandong, à la frontière coréenne) et Moukden (capitale provinciale chinoise à michemin entre Dalian et Changchun, plus grande ville de la région, actuelle Shenyang) pour en faire une ligne véritable (de $275 \mathrm{~km}$ ) faisant la jonction entre les lignes sudmandchourienne et transcoréenne. Un pont ferroviaire de près d'un kilomètre sur le fleuve Yalu relia les deux postes-frontière d'Andong et Sin-ŭiju en 1911. En 1912 puis 1914, les lignes conduisant à Mokp'o et Wŏsan étaient achevées. Long de 1220 km 
en $1918^{29}$, le réseau ferré aménagé par le Japon répondait d'abord à des objectifs militaires avec un tracé portant sur le front Nord, la Mandchourie et la Russie.

Voyons maintenant les résultats comptables du réseau ferroviaire colonial coréen, tels que présentés par le gouvernement général.

Si les bilans comptables du chemin de fer coréen affichèrent toujours des bénéfices, cela ne signifie pas pour autant que l'entreprise le fût vraiment: la marge dégagée en fin d'année servait notamment à payer les crédits contractés pour financer les travaux d'aménagement ${ }^{30}$. Le tracé militaire du réseau n'était par ailleurs pas nécessairement compatible avec le développement économique du réseau ${ }^{31}$ : il se limitait à un axe pénétrant du sud vers le nord sur lequel devaient se fixer les lignes auxiliaires, rendant à terme inévitable une saturation logistique. Compte tenu des paiements de crédits, seule l'année 1917 semble avoir été véritablement bénéficiaire : le volume et les recettes du trafic marchandises augmentèrent respectivement de $30 \%$ et $38 \%{ }^{32}$. La demande pour le transport ferroviaire avait été grandement stimulée par le manque de navires marchands dû à la guerre en Europe (en 1916 déjà le déficit avait presque été réduit à néant) ${ }^{33}$.

Comme les graphiques I et II le montrent, l'activité du trafic-marchandises du chemin de fer coréen était très diversifiée, à l'inverse d'une ligne ferroviaire coloniale classique mono-orientée. La diversité était telle que la catégorie statistique des "divers autres » dépassa même celle des produits agricoles entre 1918 et 1922. Produits agricoles et minerais représentaient à eux seuls la majorité du trafic (53\%) entre 1910 et 1920. L'article le plus important était le charbon, représentant à lui seul près d'un quart du volume (23\%). Combustible indispensable au fonctionnement du chemin de fer luimême, il fallait en importer du Japon et de Mandchourie (les trois quarts du volume consommé en Corée en 1918-20) ${ }^{34}$. Si l'on ajoute aux produits agricoles les produits forestiers et ceux de la mer, la part du secteur primaire au sein des catégories identifiées est écrasante $(90 \%)$. Les $10 \%$ restants revenaient au secteur des produits finis (kōsan-hin 工産品). Le riz ne réalisa pas de croissance particulièrement importante, au contraire, nous voyons qu'avec la guerre, le volume transporté stagna alors que celui des autres denrées agricoles (soja, blé, maïs, etc.) augmenta continuellement. En termes de volume (en tonnes) la croissance fut soutenue, mais en dents de scie: les fortes poussées de 1910, 1912 et 1914-17 (+22\% en moyenne) furent toutes suivies de taux presque nuls $(1911,1913,1918 ;+3 \%$ en moyenne). Cette instabilité alla s'aggravant en fin de période : aux records de 1917 (+30\%) et 1919 (+40\%) correspondit la forte chute de 1920 (-13\%). La croissance en question était profondément instable, participant d'une surchauffe. Cette instabilité rongea les bénéfices (graph. III): après avoir cru fortement jusqu'en 1917, ils plongèrent lourdement entre 1918 et 1920 . La croissance du trafic de marchandises a marqué l'ensemble de la période, mais elle atteignit clairement son pic entre 1917 et 1919 (+25\%; contre $+11 \%$ entre 1914 et 1916$)$ avant le contrecoup de 1920 (-13\%). Le flux des voyageurs montre avec plus de force encore la rupture de 1917 : sur cette année le nombre de passagers passa de 240000 à 350 000. Les bénéfices affichés (graph. III) sont hors frais d'investissements (pris en charge par l'administration coloniale), sans quoi ils n'eurent point existé. Le graphique dessine bien la dynamique en action: une croissance intensive, suivie d'une chute plus rapide encore... alors même que les volumes transportés (marchandises et, surtout, voyageurs) continuaient de croître (graph. III). 
Graph. I: Le trafic marchandises du chemin de fer coréen, en yens (1910-1922)

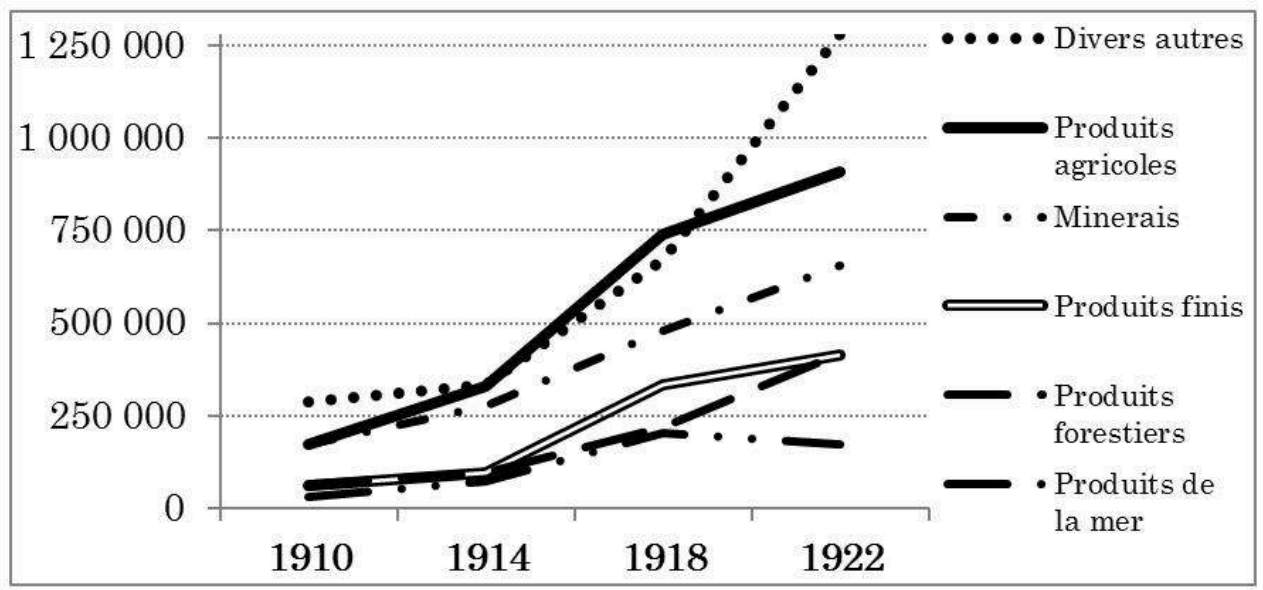

Source : Chōsen sōtokufu tetsudō-kyoku 朝鮮総督府鉄道局, Chōsen tetsudō yonjū-nen ryakushi 朝鮮鉄 道四拾年略史 (Histoire des Quarante ans du Chemin de fer en Corée), Keijō, 1940, p. 568

Graph. II : Le trafic marchandises du chemin de fer coréen, en tonnes (1910-1290)

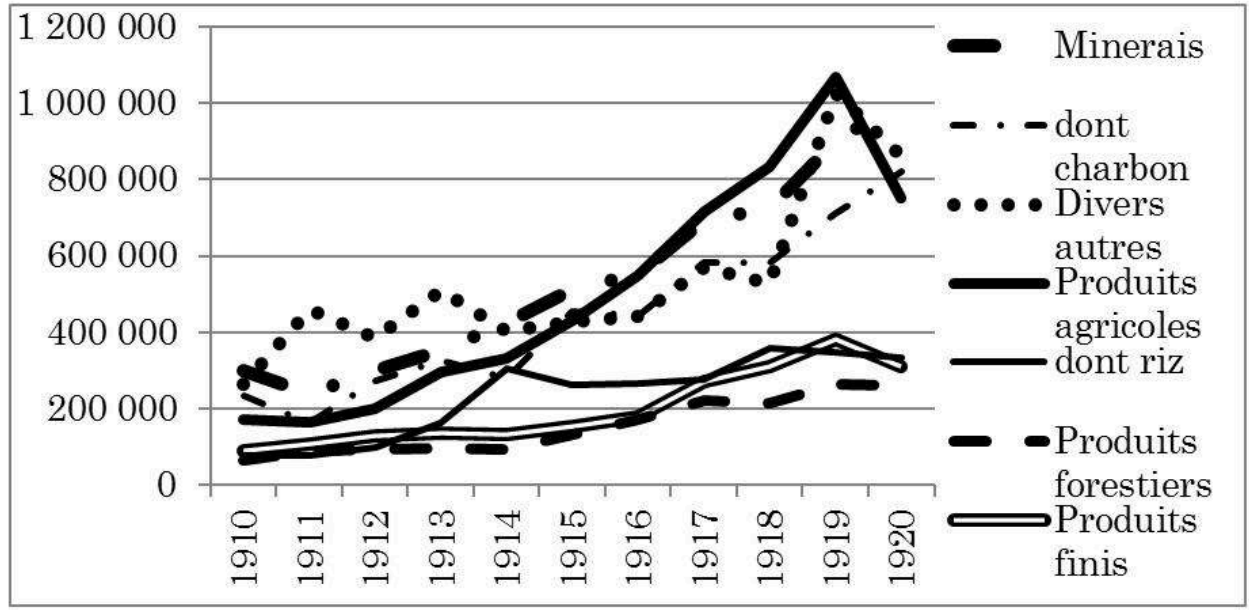

Source : Chōsen sōtoku-fu tetsudō-kyoku, Chōsen tetsudō ronsan, op. cit., p. 163. 
Graph. III : L'évolution indiciaire des activités et des bénéfices du chemin de fer coréen

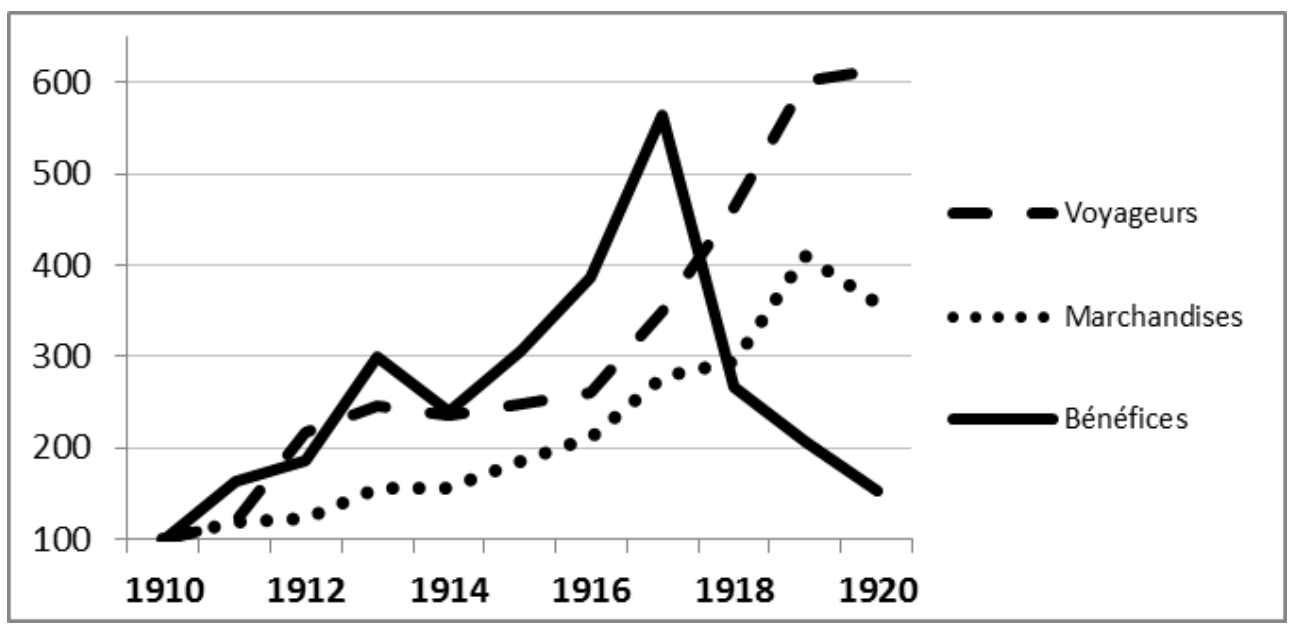

N.B. : Les données-source « voyageurs » sont en nombre de personnes transportées, les «marchandises » en tonnes, les bénéfices en yens (moins les dépenses de fonctionnement sans déduction du service de la dette).

Source : Chōsen sōtoku-fu tetsudō-kyoku, Chōsen tetsudō ronsan, op. cit., p. 375-375, p. 171.

Nous avons rapidement survolé les voies terrestres de communication, venons-en à la question des mouvements financiers de la colonie.

\section{Les finances coloniales}

16 Nous avons déjà vu que le capitalisme japonais n'était pas assez puissant à l'aube du $\mathrm{xx}^{\mathrm{e}}$ siècle pour avoir motivé la constitution de colonies. De fait, la documentation produite par le gouvernement général de Corée, après 1910, nous explique que sous le régime du protectorat en Corée, les dépenses administratives de Tōkyō en plus des travaux ferroviaires coûtèrent cher : quelque cent quatre millions de yens entre 1907 et 1910 seulement ${ }^{35}$. Quoique l'on ne puisse mesurer l'activité économique réelle de cette façon, il faut noter que le passage au régime de l'administration directe en 1910 a permis de refonder le budget des autorités et de le restructurer entièrement autour du développement de la domination coloniale. Ce que montre le tableau I. 
Tabl. I : La situation financière des administrations coloniales japonaises (1907-1918) (unité : milliers de yens)

(unité : milliers de yens)

\begin{tabular}{|c|c|c|c|c|c|c|c|c|c|c|c|c|}
\hline & \multicolumn{4}{|c|}{ KWANTUNG } & \multirow{2}{*}{$\begin{array}{l}\text { TAIWAN } \\
\text { (D) }\end{array}$} & \multicolumn{2}{|c|}{ COREE } & \multirow[b]{5}{*}{ (B) } & \multirow[b]{5}{*}{ (D) } & \multirow[b]{5}{*}{ S/D } & \multirow[b]{5}{*}{ SA/D-S } & \multirow[b]{5}{*}{$\mathrm{SA} / \mathrm{DA}$} \\
\hline & (B) & (D) & (S) & $(\mathrm{S} / \mathrm{D})$ & & (A) & (S) & & & & & \\
\hline 1907 & -2179 & 3472,5 & 3001 & $(86 \%)$ & 27710 & 10626 & 16374 & & & & & \\
\hline 1908 & -156 & 115 & 40,5 & $5(35 \%)$ & 30666 & 15229 & 15771 & & & & & \\
\hline 1909 & -1298 & 4618 & 2964 & $(64 \%)$ & 30189 & 10358 & 15642 & & & & & \\
\hline 1910 & -1437 & 5771 & 3615 & $(63 \%)$ & 41202 & $\begin{array}{lll}10 & 193\end{array}$ & 25000 & -849 & 18257 & $29 \%$ & $85 \%$ & $54 \%$ \\
\hline 1911 & -1623 & 5499 & 3692 & $(58 \%)$ & 43621 & 9652 & 12350 & -12347 & 48742 & $25 \%$ & $45 \%$ & $38 \%$ \\
\hline 1912 & -1733 & $5359,5 ?$ & 3125 & (53\%) & 47189 & 8984 & 12350 & -11586 & 52892 & $23 \%$ & $40 \%$ & $34 \%$ \\
\hline 1913 & -1332 & 4429 & 2347 & $(54 \%)$ & 44474 & 8233 & 10000 & -10343 & 57990 & $17 \%$ & $31 \%$ & $28 \%$ \\
\hline 1914 & -1437 & 4117 & 2233 & $(47 \%)$ & 47696 & 7069 & 9000 & -5586 & 56413 & $16 \%$ & $28 \%$ & $25 \%$ \\
\hline 1915 & -1340 & 4119 & 1937 & $(48 \%)$ & 38250 & 6971 & 8000 & -3996 & 58873 & $14 \%$ & $25 \%$ & $23 \%$ \\
\hline 1916 & -842 & 4208 & 2007 & (41\%) & 42687 & 8737 & 7000 & -2512 & 59849 & $12 \%$ & $26 \%$ & $23 \%$ \\
\hline 1917 & -444 & 4936,5 & 2007 & $(42 \%)$ & 46167 & 10536 & 5000 & -527 & 62643 & $8 \%$ & $25 \%$ & $21 \%$ \\
\hline 1918 & 21 & 6553 & 2007 & $(31 \%)$ & 55335 & 11189 & 3000 & -3322 & 65142 & $5 \%$ & $22 \%$ & $19 \%$ \\
\hline 1919 & 118 & 11706 & 2106 & $(18 \%)$ & 72323 & 15383 & 0 & 12944 & 77561 & $0 \%$ & $20 \%$ & $17 \%$ \\
\hline 1920 & 557 & 13793 & 3000 & $(22 \%)$ & 95334 & 17857 & 10000 & -13862 & 114317 & $1 \%$ & $24 \%$ & $21 \%$ \\
\hline
\end{tabular}

(B) : balance réelle du budget civil ordinaire (recettes ordinaires - subventions métropolitaines dépenses ordinaires)

(D) : dépenses civiles totales (ordinaires et extraordinaires)

(s) : subventions métropolitaines (incluant pour le Kwantung les « versements » hokyū-kin 補給金de la $\mathrm{C}^{\mathrm{ie}} \mathrm{du}$ Chemin de fer sud-mandchourien pour l'administration des terres longeant ses rails (mantetsu fuzoku-chi 満鉄附属地).

(A) : dépenses militaires (métropole)

(sA) : dépenses métropolitaines (subventions+ armée) ; D-s : dépenses péninsulaires (dépenses subventions); DA : total des frais (dépenses + armée)

*) Kwantung : terres adjacentes au chemin de fer gérées par la CCSM comprises, et seulement elles en 1908.

POUR LA CORÉE : Government-General of Chosen, Annual Report on Reforms and Progress in Chosen, Keijō, 1922, p. 37 ; 1910 : mizoguchi Toshiyuki 溝口敏行, Taiwan Chōsen no keizai seichō 台湾・朝鮮経 済の成長 (La croissance économique de Taiwan et la Corée), Iwanami shoten, 1975, 180 p., p. 129. Subventions, 1907-1910 : voir Government-General of Chosen, Results of Three Years' Administration of Chosen since Annexation, 97 p., p. 17. Sous le régime du résident général entre 1907 et 1910, la métropole subvenait entièrement aux dépenses de l'administration coloniale. Pour 1910, avec la création du Gouvernement général, les subventions métropolitaines ne furent que de 5291000 de yens selon Kimura (voir ci-après). Le gouvernement général explique (voir la source précédente) qu'elles furent de vingt-cinq millions de yens aide dépenses militaires comprises. Peut-être peut-on expliquer les cinq millions de yens évoqués par Kimura par le financement péninsulaire de l'administration coloniale (manifestement, sur cette première année le financement a été transitoire, comparé aux années suivantes il a par ailleurs été moitié moindre). Subventions, 1911 : kimura Mitsuhiko, "The Public Finance in Korea under Japanese Colonial Rule : Deficit in the Colonial Account and Colonial Taxation", Explorations in Economic History, vol. 26, n³, 1989, p. 285-310, p. 288.

POUR LE KWANTUNg: South Manchuria Railway, Report on Progress in Manchuria, Dairen, 1929, 6. vol. vol. 1, 264 p., p. 57; Manmō bunka kyōkai 満蒙文化協会 (Association culturelle de Mandchourie et Mongolie), Manmō nenkan 満蒙年鑑 (Annuaire [statistique] de Mandchourie et Mongolie), Dairen, année 1924, p. 206-207, p. 216-217.

PouR talwaN : mizoguchi T., Taiwan Chōsen..., op. cit., p. 129. Pour les dépenses militaires («Armée ») en Corée: Hojin ch'oe, Essays on Korean Economy, Séoul, Sekyungsa, 1995, 238 p., p. 185.

Pour le Kwantung (Mandchourie) et la Corée, si la forte baisse de la dépendance envers les subventions est indéniable, les montants en jeu restèrent importants. Le tableau I montre une amélioration des finances coréennes telle qu'on pourrait croire à la conquête, bien que longue et difficile, d'une autonomie financière en fin de période, au moment même où les bénéfices des chemins de fer s'effondraient. Or, comme nous le montre la balance budgétaire ordinaire réelle (B), l'instabilité et le déficit étaient structurels, de sorte que l'arrêt des subventions en 1919 à la faveur d'importantes 
recettes fiscales ordinaires fut aussi symbolique que temporaire. Cette augmentation des recettes fiscales résidait à $85 \%$ dans la croissance des recettes ordinaires ${ }^{36}$ : les revenus des douanes étaient en augmentation de 28 \% (grâce au bond des importations, $+75 \%$ ) et cela malgré l'abolition de la taxe à l'exportation vers la métropole (ishutsu-zei

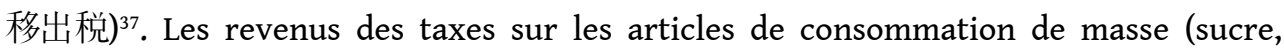
opium, tabac, alcool, imprimés) ont apporté une plus-value importante ( $+3,7$ millions de yens). Différents autres revenus publics ont aussi apporté leur pierre, comme ceux de l'exploitation forestière, la contribution de la Compagnie du chemin de fer sudmandchourien au titre de sa gestion des chemins de fer coréens, ou encore le travail carcéral (+3 millions de yens). La plus-value fiscale de 1919 s'est donc faite "par le bas", par la taxation des biens de consommation de masse (importés ou non), aggravant la pression inflationniste sur le pouvoir d'achat des masses colonisées.

Une des raisons de l'effacement des subventions métropolitaines était qu'en réalité la métropole ne donnait plus, mais vendait son argent à l'administration coloniale. Cette dernière contracta des emprunts toujours plus importants, assurant une rente permanente aux souscripteurs japonais, point sur lequel il faudrait se pencher davantage pour analyser l'expropriation coloniale. Concrètement: en 1910, l'argent parti vers la colonie coréenne pour revenir vers les investisseurs s'élevait déjà à un montant de plus de vingt et un millions de yens, sous le poids financier de l'aménagement des infrastructures de transport et communication (en premier lieu, le chemin de fer). Le budget ordinaire ne pouvant pas régler de telles dépenses, le plafond de ses emprunts fut quadruplé entre 1915 et 1920 (de 56 millions à 206,5 millions de yens) ${ }^{38}$, ce qui représentait $161 \%$ des recettes propres (hors subventions métropolitaines) de l'administration coloniale en 1918. Ce taux baissa légèrement en 1919 (159\%), puis surtout en 1920 (119\%), ce qui était surtout dû à l'inflation démesurée d'alors et à des mesures tout aussi exceptionnelles que factices (augmentation des recettes fiscales, retour des subventions métropolitaines, lancement de nouveaux emprunts publics) servant surtout à payer les intérêts des investisseurs, et, d'après le gouvernement général de Corée, à dégonfler provisoirement la « dette " globale $^{39}$. Remplir le tonneau percé des finances coloniales coréennes était lucratif pour la métropole, c'était aussi et surtout un moyen supplémentaire de dominer la péninsule.

Un dernier élément pour conclure sur les finances publiques en Corée: le coût d'entretien des garnisons militaires (près de 18 millions de yens sur les deux seules années 1916-17, financé directement par la métropole ${ }^{40}$. Cette somme importante pourrait être interprétée comme une injection d'argent en Corée, mais dans la mesure où les soldats étaient métropolitains et que l'armée dépensait surtout en liaison avec des entreprises métropolitaines, la prise en compte de ce chiffre dans le calcul des pertes et gains au sein du rapport colonial nous semble peu pertinente. Malgré tout, subventions administratives et dépenses militaires correspondaient à une part très importante et stable de l'ensemble des dépenses coloniales (un quart, $25 \%$, entre 1913 et 1920). Il est donc, évidemment, impossible de parler d'« autonomie " financière de la colonie sur notre période, la Corée étant, comme on le voit, soumise au pouvoir économique de la métropole. Il faudra analyser la question des échanges commerciaux extérieurs de la péninsule sous cet angle. 
Graph. IV : La balance commerciale coréenne (1910-1920) (unité : milliers de yens)

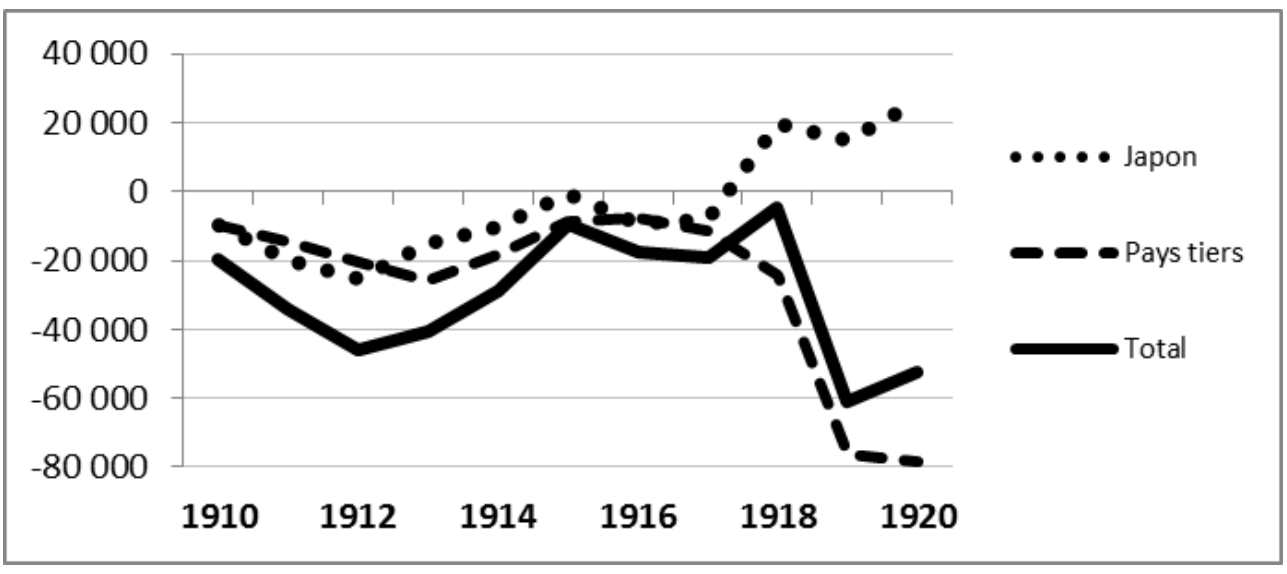

Source : yamamoto Yūzō, Shokuminchi keizai-shi kenkyū, op. cit., p. 167.

Sur l'ensemble de la période, le mouvement commercial extérieur de la Corée a été largement déficitaire (graph. IV), à hauteur de trente millions de yens par an en moyenne (soit un taux de $19 \%$ ) $^{41}$. Avec la métropole, le déficit atteignait soixante-neuf millions de yens sur la période 1912-1917, tandis que les subventions versées dans le sens inverse n'étaient que de cinquante et un millions de yens. Ce déficit vis-à-vis de la métropole alla toutefois diminuant alors qu'il représentait la quasi-totalité de la « dette commerciale » du pouvoir colonial de Corée sur 1910-1912. Ce basculement «dans le positif » était dû au soudain accroissement des importations japonaises de riz coréen à la fin de la Première Guerre mondiale et de la baisse des importations coréennes, la péninsule n'ayant pas les moyens de suivre la hausse des prix en métropole, deux phénomènes négatifs pour le peuple coréen.

Dans ce contexte, la métropole accorda moins de moins en moins de subsides au fur et à mesure que son excédent commercial avec la Corée diminuait, veillant à ne pas » donner » plus qu'elle ne recevait. Le déficit commercial global de la colonie a ainsi constitué une charge supplémentaire pour l'économie péninsulaire.

Venons-en maintenant aux institutions financières de la péninsule sur cette période.

\section{De la Banque $\mathrm{n}^{\circ} 1$ à la Banque coloniale de Corée}

En dehors de l'infrastructure ferroviaire et de la structure administrative, la seule institution japonaise d'importance établie en Corée dès les débuts de la colonisation était la Banque $\mathrm{n}^{\circ} 1$ (Dai-ichi ginkō 第一銀行) de Shibusawa Eiichi. C'était même l'entreprise moderne la plus anciennement établie sur place puisqu'elle s'établit en Corée dès 1878 à la demande de Tōkyō pour développer le commerce nippo-coréen et accroître la présence japonaise sur la péninsule ${ }^{42}$. L'« incident de Kanghwa » (1875) s'était conclu à la faveur du Japon, qui réussit à imposer l'ouverture officielle du commerce bilatéral (traité de Kanghwa,1876). L'activité de la Banque $\mathrm{n}^{\circ} 1$ se limita d'abord au financement des opérations commerciales (gérant même les Douanes coréennes à partir de 1883) ${ }^{43}$ avant de servir à l'intendance des troupes de l'archipel durant la guerre sino-japonaise. Pendant la guerre, les dépenses militaires créèrent un influx de yens sur la péninsule alors que le royaume coréen éprouvait déjà de sérieuses difficultés avec son système de multiples monnaies plus ou moins anciennes (il avait 
entamé une politique de fuite en avant en multipliant la frappe monétaire, notamment avec de nouvelles pièces de nickel, véritable "monnaie de singe ») ${ }^{44}$. L'imbroglio des pressions japonaises puis russes aggrava la situation : malgré l'adoption de l'étalon-or par le Japon en 1897, ce dernier obtint le maintien de la circulation en Corée de ses yens-argent (le yen-or s'imposerait de toute manière dans le cadre du commerce bilatéral)... Mais sous la pression russe, Séoul fit volte-face en 1901 et interdit la chose pour émettre (à partir de 1902) sa propre monnaie or ${ }^{45}$. Or, au même moment, la Banque $\mathrm{n}^{\circ} 1$ se voyait autorisée par le pouvoir coréen, après lui avoir accordé un prêt ${ }^{46}$, à émettre ses propres billets, adossés au yen-or ${ }^{47}$, c'est-à-dire assurant la diffusion de ce dernier contre la toute nouvelle monnaie-or nationale. Cette monnaie de la Banque $\mathrm{n}^{\circ} 1$ devint la monnaie officielle ${ }^{48}$ sous le régime du protectorat, jusqu'à l'adoption formelle du yen en 1910. On procéda jusqu'en 1920 au retrait progressif des monnaies coréennes d'or et d'argent (une campagne jonchée de tromperies sur les différents taux d'échange, notamment de la part des usuriers ${ }^{49}$. Dans les faits, ces monnaies argent et (surtout) or restaient réservées aux transactions importantes. Le peuple, lui, faisait surtout usage de petites monnaies en cuivre. Très populaire et ayant une très faible valeur d'échange face à l'or, le pouvoir japonais décida de ne pas en forcer le retrait pour seulement en limiter l'usage légal aux sommes inférieures à un yen ${ }^{50}$.

Avec la construction d'une administration coloniale, le besoin d'une banque centrale effectivement basée en Corée poussa à la création en 1909 de la Banque de Corée (kankoku ginkō 韓国銀行). Elle reprit les actifs coréens de la Banque $\mathrm{n}^{\circ} 1$ (devant lui rembourser près de huit millions de yens sur vingt ans sans intérêts) ${ }^{51}$ et devint en 1910 la Banque coloniale de Corée (chōsen ginkō 朝鮮銀行, BCC dorénavant) ${ }^{52}$. Banque centrale, la BCC fut loin de se limiter au rôle d'émetteur monétaire : elle poursuivit les affaires commerciales de la Banque $\mathrm{n}^{0} 1$ et ce double travail porta sur d'immenses volumes monétaires. La persistance des monnaies antérieures et l'existence d'institutions traditionnelles de crédit amenaient déjà la BCC à avancer de larges fonds pour les absorber et, les échanges commerciaux extérieurs imposés à la péninsule étant lourdement déficitaires, il aurait théoriquement aussi fallu soutenir les exportations et la naissance de la production industrielle. Cela laissa de la place à un certain nombre de banques commerciales qui se sont développées dans les années 1910, déployant un réseau de cinquante-huit bureaux en $1919^{53}$. Elles semblent avoir constitué une aide importante au commerce intérieur, mais internationalement, seule la BCC avait les moyens de peser véritablement. Elle n'ouvrit d'ailleurs de nouveaux bureaux qu'en dehors de la péninsule. Sa terre d'expansion favorite était la Mandchourie : en 1918, elle y possédait même plus de bureaux qu'en Corée (seize contre douze) $)^{54}$. Ce réseau était complété par deux bureaux ailleurs en Chine (Shanghai et Qingdao) et trois métropolitains (Tōkyō, Kōbe, Ōsaka). La BCC possédait alors trente-trois antennes sur toute l'Asie du Nord-Est. Ce « tournant mandchourien » fut opéré brusquement à partir de 1916 : les bureaux mandchouriens ne détenaient que $6 \%$ des dépôts de la banque entre 1910 et 1914 contre quatre fois plus ( $21 \%$ ) entre 1916 et 1919 , et $5 \%$ des prêts entre 1910 et 1915 contre $28 \%$ entre 1916 et 1919. En parallèle, les dépôts et prêts en métropole ont bondi : de $4 \%$ des dépôts en 1915 à $61 \%$ entre 1917 et 1919, de $7 \%$ des prêts entre 1910 et 1914 à $44 \%$ entre 1917 et $1919^{55}$. Ainsi la part coréenne ne dépassait plus celle des bureaux mandchouriens en 1918 et 1919.

À la fin de la période, les activités péninsulaires de la $\mathrm{BCC}$, bien qu'elle fût banque centrale de Corée, étaient devenues minoritaires. La BCC elle-même fuyait l'économie 
coloniale moribonde qu'elle était censée dynamiser... à partir de 1915, cette « fuite » est évidente, mais il faut remarquer que la terre d'accueil a d'abord été la métropole, en demande de capitaux pour répondre au «boum » économique de la Grande Guerre. La BCC ne s'est transformée en conduit du capital japonais vers la Mandchourie qu'après cela, en 1916, à partir de l'accession au pouvoir à Tōkyō de Terauchi Masatake 寺内正 毅, le premier gouverneur général de Corée (1910-1916), maintenant Premier ministre. L'histoire de la BCC offre ainsi de larges possibilités d'étude, à explorer.

Graph. v : La géographie des dépôts de la Banque coloniale de Corée (1910-1919)

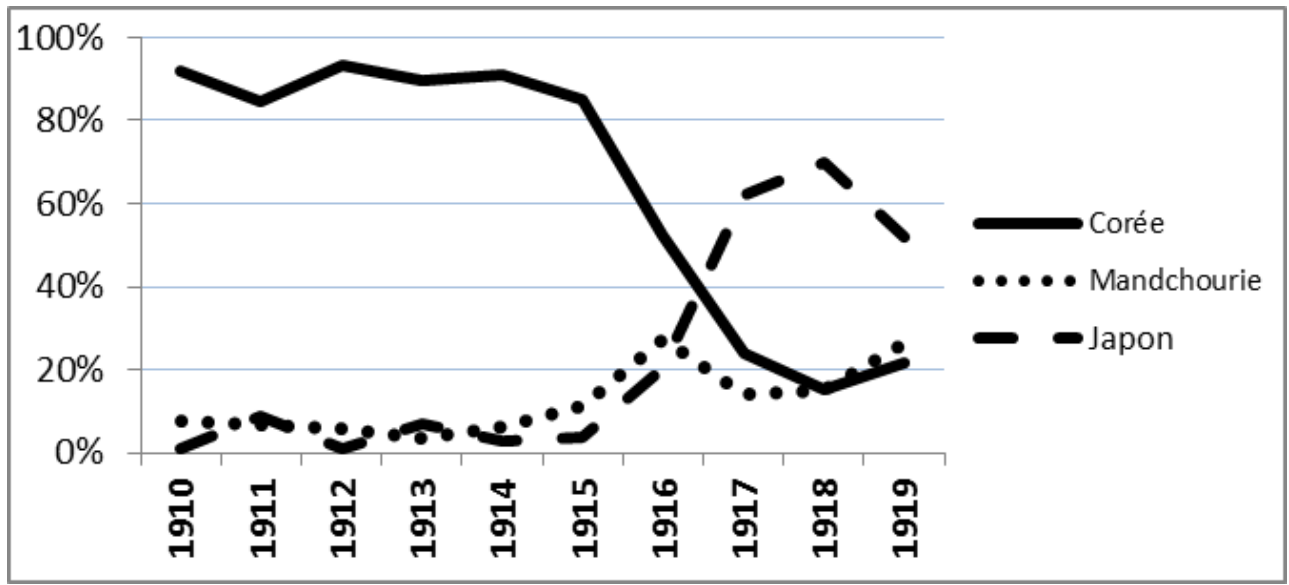

SOURCE : BANK OF CHOSEN, ECONOMIC HISTORY OF CHOSEN, OP. CIT., P. 179.

Graph. vI : La géographie des prêts de la Banque coloniale de Corée (1910-1919)

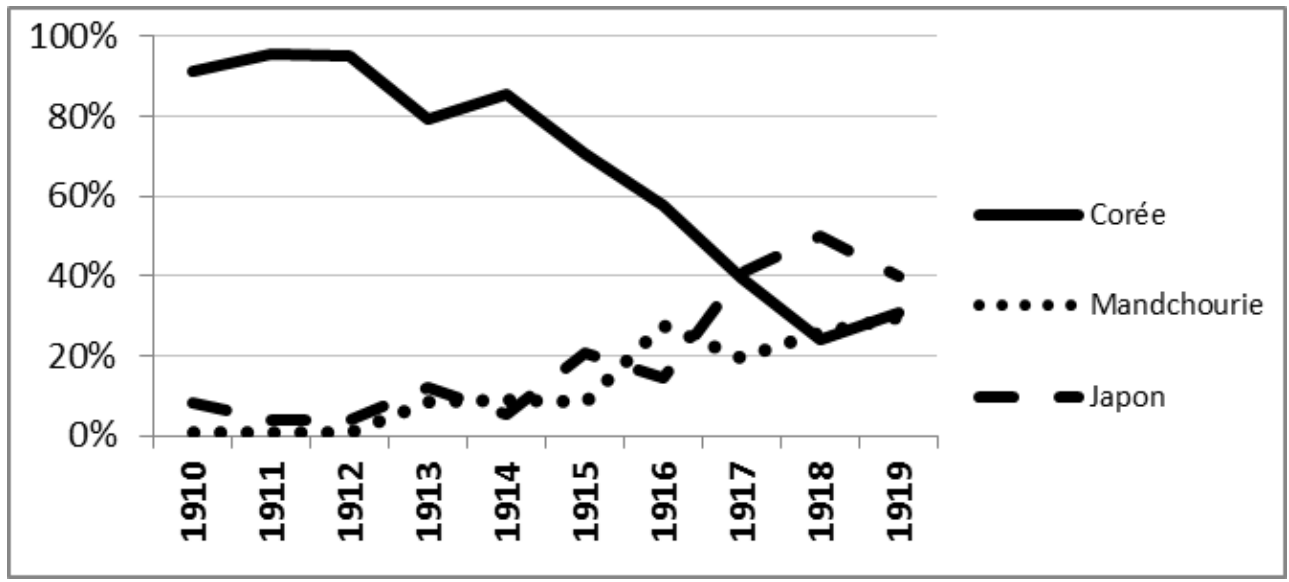

Source : Idem.

Que ce fût pour le surplus de capital à investir ou l'importance même de ces investissements pour l'industrie japonaise, soulignons ici simplement que cette dynamique prenait appui sur l'emballement économique en métropole. On ne soulignera donc jamais assez l'importance de bien saisir la bulle financière de la Grande Guerre pour analyser l'histoire du développement économique et industriel en Corée, ainsi que le tournant mandchourien de la politique extérieure japonaise vers cette même période. 
Venons-en maintenant à un autre aspect central des politiques économiques japonaises en Corée : les secteurs économiques productifs.

\section{Les structures de la production : entreprises et « industrie »}

Graph. VII : La valeur de la production économique matérielle coréenne par secteurs (1910-1920) (milliers de yens)

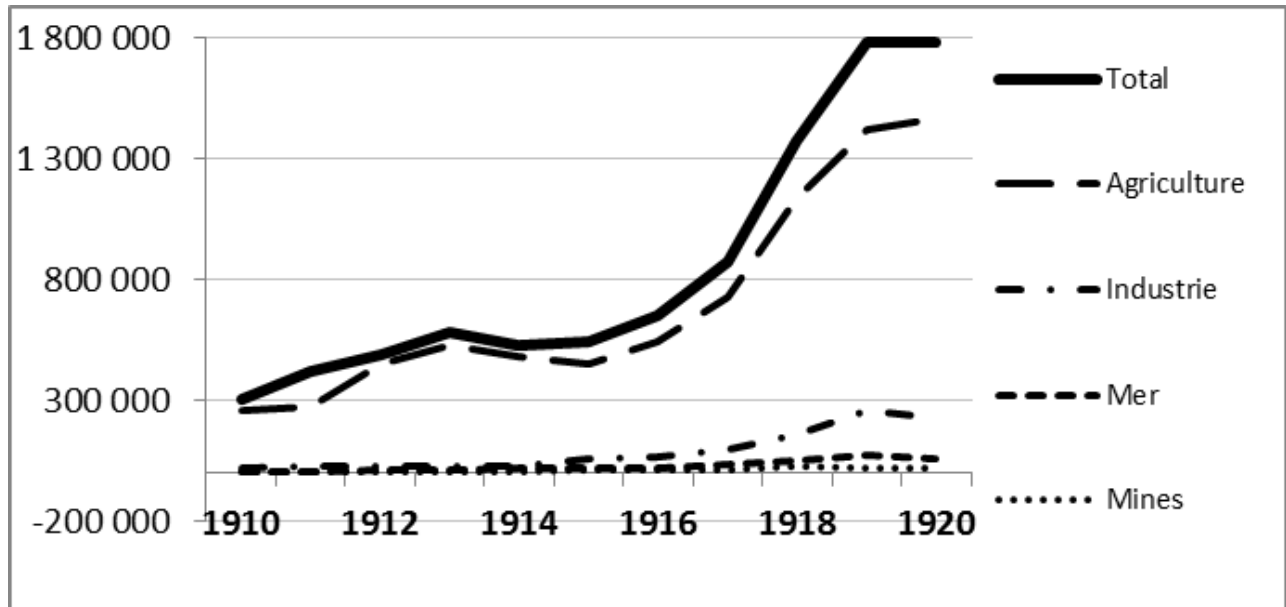

Source : Chōsen sōtokufu tetsudō-kyoku, Chōsen tetsudō ronsan, op. cit., p. 143

Comme le montre le graphique vII, établi ici à partir des données présentées par le gouvernement général et concordant avec l'historiographie d'une manière générale, l'agriculture représentait tout au long de la période la quasi-totalité $(85 \%)$ de la production économique matérielle coréenne. Entre 1918 et 1920, cette part baissa à $80 \%$, ce qui s'explique par la montée de la production industrielle. Selon l'estimation faite par le collectif dirigé par Kim Nak Nyeon, l'agriculture comptait pour $68 \%$ du produit national brut entre 1911 et 1913 et $65 \%$ en 1920, les services comptant pour $25 \%$ du total en 1911 et $27 \%$ en $1919^{56}$. Manifestement, la période a connu des changements importants en termes de production économique, pour tenter d'appréhender la réalité de ces changements, nous allons d'abord analyser le capital des entreprises coloniales puis les différents secteurs d'activité concernés.

\section{Les entreprises coloniales, une approche par le capital}

Pour comprendre la réalité et la dynamique du tissu des entreprises coloniales, nous allons présenter et analyser une série de données statistiques portant sur la propriété et la localisation du capital entre 1910 et 1920 , puis sur sa distribution par secteurs d'activité entre 1914 et 1920. Ayons à l'esprit que les données officielles sont imparfaites, et que l'approche suivie ici ne permet pas nécessairement de saisir l'ensemble des entreprises et notamment les plus petites constituant le tissu économique coréen. Par ailleurs, nous intéressant au phénomène colonial, nous ne traiterons pas des entreprises étrangères. La raison en est que ces dernières constituaient des structures très particulières: bien que très peu nombreuses (dix en 1910), leur poids en capital dépassait celui des entreprises coréennes. Cela s'explique 
notamment par le fait que parmi elles figuraient les représentations d'entreprises comme la Standard Oil Company du multimilliardaire John D. Rockfeller. Elles étaient engagées dans le commerce et les mines, surtout les mines d'or dont elles tenaient la quasi-totalité de la production (notamment l'américaine Oriental Consolidated Mining Company). Sur la période 1905-1915, l'or était le principal minerai extrait en Corée (69\% de l'exploitation minière), mais il fut rapidement dépassé par l'exploitation ferrifère et houillère (14,5\% de la production minière en 1919-20) ${ }^{57}$. Cette propriété étrangère et cet effacement relatif assez rapide expliquent l'absence d'attention particulière pour cette question de l'or dans l'historiographie et le fait que nous ne rentrerons nous-mêmes pas plus dans les détails ici. Commençons par observer l'évolution globale entre 1910 et 1920 des entreprises selon qu'elles avaient leur siège en Corée (entreprises péninsulaires) ou en métropole (entreprises métropolitaines).

Graph. VIII : Le nombre des entreprises péninsulaires (1910-1920)

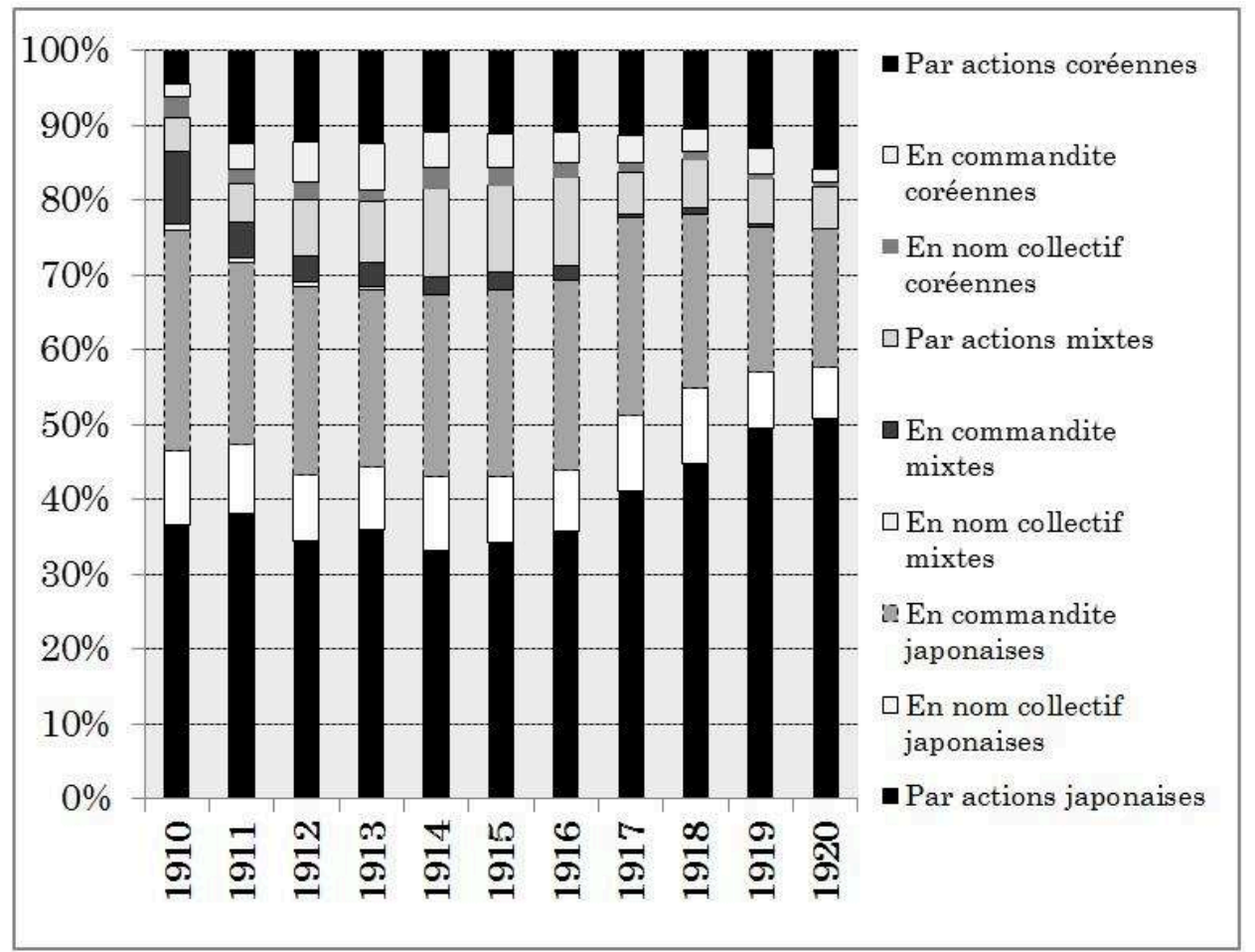

$\underline{N}$.B. : entreprises étrangères au siège social basé en Corée comprises (1 entre 1912-19 et 2 en 1920). Source : CSTN, année 1920, t. 3. 


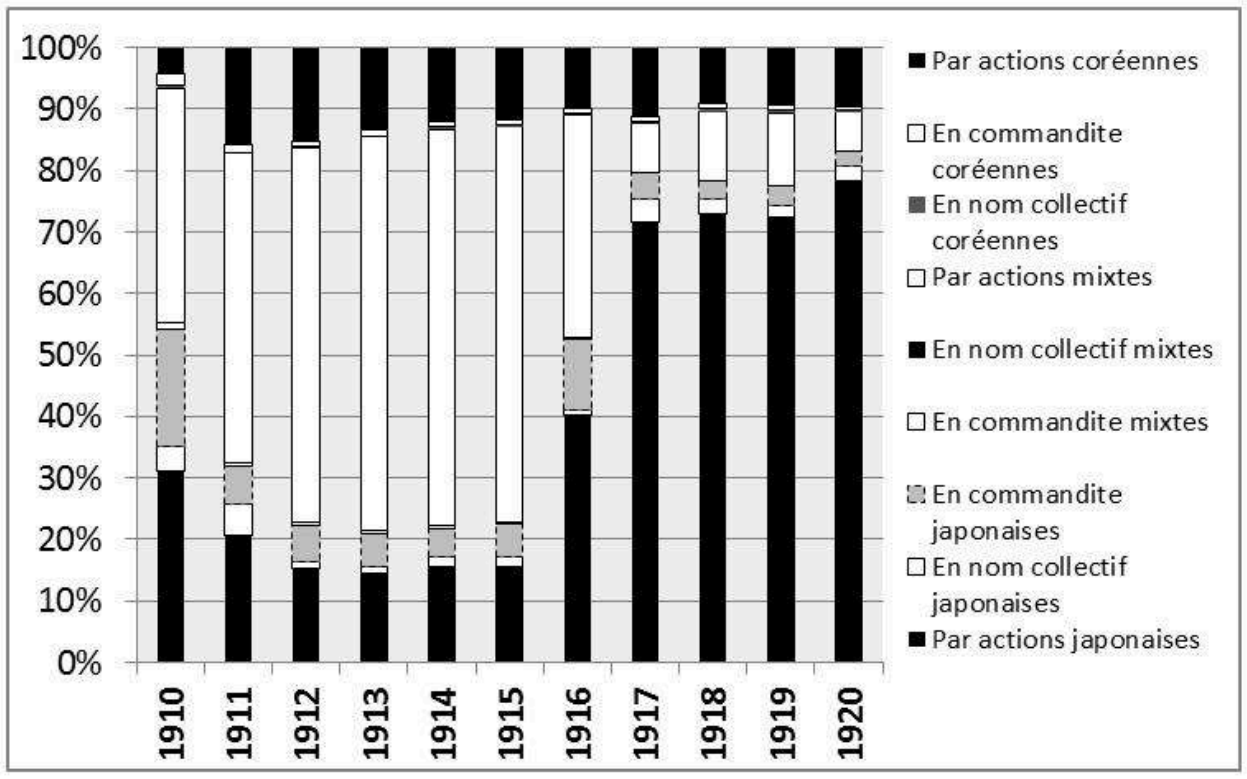

Source : CSTN, année 1920, t. 3. péninsulaires selon la nationalité des propriétaires, par leur nombre (voir le graph. VIII) et leur montant en capital versé (voir le graph. Ix). De manière générale, on remarque d'abord un décalage entre une structure multipolaire des entreprises vues par leur nombre et une structure hégémonique dans la répartition du capital. La multipolarité structurelle des entreprises s'est toutefois amenuisée avec la montée en puissance des entreprises par actions japonaises à partir de 1917 (un tiers des entreprises péninsulaires entre 1911 et 1916, la moitié en 1919 et 1920). Les sociétés par actions, coréennes et mixtes (gōdō 合同) incluses, gagnèrent ainsi dix points sur l'ensemble pour en occuper les deux tiers en 1918-20 (contre $57 \%$ entre 1911 et 1916). D'autre part, les sociétés en commandite représentaient un tiers du total entre 1911 et 1913 et ne perdirent que deux points pendant la guerre (31\% entre 1914 et 1917), mais chutèrent jusqu'à $20 \%$ en 1920. Enfin, la part des sociétés en nom collectif resta stable (à $11 \%$ ). En termes de catégorisation "nationale», la chute des entreprises mixtes est impressionnante : après avoir gagné trois points entre 1911 et 1916, leur part fondit de plus de moitié sur la seule année 1917 (de $14 \%$ à $6 \%$ ). Ce sont les entreprises japonaises qui les ont supplantées, leur part du total passant de $69 \%$ à $77 \%$ entre 1911-16 et 1917-20. Placées entre ces deux, les entreprises coréennes occupèrent une part stable (à $18 \%$ ). Encore faut-il se demander si cette compression à partir de 1916 de la catégorie mixte sous l'effet du développement des sociétés par actions japonaises se retrouve ou non dans la répartition du capital.

En termes de capitaux (graph. Ix), en suivant toujours les informations officielles (qui mesuraient néanmoins sans doute plus précisément les entreprises de métropole), non seulement cette tendance est confirmée, mais elle est aussi précisée : le capital boursier dont disposaient les entreprises mixtes a été laminé à partir de 1916, chutant en 1917 brutalement de plus d'un tiers (36\%). Le montant perdu (neuf millions de yens) se retrouvait dans l'augmentation cette même année du capital des sociétés par actions japonaises (triplant de volume). Ce «siphonage» continua en 1917 : tandis que les 
entreprises par actions mixtes perdaient onze millions de yens (provoquant une hécatombe : douze unités sur vingt-cinq furent liquidées) leurs homologues japonaises doublaient encore leur capital et portaient leur nombre de 76 à 94 . Comme on le voit sur le graphique Ix, leur part dans le capital péninsulaire bondit de $16 \%$ à $72 \%$ en deux ans. Dans ce mouvement, la part coréenne fut réduite de $17 \%$ en 1911 à $11 \%$ en 1920. Parmi les entreprises péninsulaires, les sociétés mixtes étaient en moyenne cinq à dix fois mieux dotées en capital que les autres jusqu'en 1915. Les moins bien loties étaient les japonaises, avant même les coréennes, signe d'une première colonisation faite de petits entrepreneurs. Cela a été bouleversé à partir de 1916 par la pénétration en force du capital boursier japonais et l'appauvrissement des structures coréennes. Or, le capital investi dans les entreprises péninsulaires était très largement boursier sur toute la période ( $90 \%$ en moyenne, chiffre dont il faut néanmoins avoir à l'esprit qu'il est aussi peut-être le mieux mesuré), c'est-à-dire que l'évolution générale a été marquée par la concentration du capital boursier sur les entreprises japonaises (recevant 99,6\% de ces nouveaux capitaux entre 1915 et 1920). Qu'en était-il des entreprises métropolitaines?

Le capital engagé dans ces dernières était sans commune mesure avec celui des entreprises péninsulaires : cinquante fois plus important, bien qu'elles fussent cinq fois moins nombreuses. Elles possédaient en moyenne douze fois plus de capitaux (entre 1911 et 1917). Selon ces données, elles ont toujours été massivement des sociétés par actions (à $98 \%$ en moyenne, avec un taux de variation de 3,4\% seulement; cf. tableau II) ; à moins que celles-ci aient été mieux repérées. Si la croissance du capital investi resta molle jusqu'en 1916, elle explosa entre 1917 et 1920 (quintuplement). Tenant compte de l'inflation, le capital entrepreneurial métropolitain en Corée doubla entre 1917 et 1920. Là encore, la force à l'œuvre était le capital boursier japonais. Sous sa pression, la part japonaise dans le total passa de $78 \%$ en 1915 à $95 \%$ entre 1917 et 1920 .

Tabl. II : Les entreprises métropolitaines (1911-1920)

\begin{tabular}{|c|c|c|c|c|c|c|c|c|c|c|c|c|}
\hline & \multicolumn{4}{|c|}{ Nombre } & \multicolumn{4}{|c|}{$\begin{array}{c}\text { Capital } \\
\text { (milliers de yens) }\end{array}$} & \multicolumn{4}{|c|}{ Moyenne } \\
\hline & n.c. & c. & a. : & Total & n.c. & c. & a. & Total & n.c. & c. & a. & Total \\
\hline 1911 & 2 & 5 & 26 & 33 & 1350 & 739 & 65506 & 67595 & 675 & 148 & 2519 & 2048 \\
\hline 1912 & 3 & 5 & $33 !$ & 41 & 1408 & 776 & $71831 !$ & 74015 & 469 & 155 & 2177 & 1805 \\
\hline 1913 & 3 & 5 & 38 & 46 & 608 & 793 & $78669 !$ & 80070 & 203 & 159 & 2107 & 1741 \\
\hline${ }^{*} 1914$ & 3 & 4 & 35 & 43 & 1058 & 637 & $84726:$ & 86421 & 353 & 159 & 2421 & 2010 \\
\hline 1915 & 2 & 4 & 37 & 43 & 1000 & 637 & $81141 !$ & 87778 & 500 & 17 & 2193 & 2041 \\
\hline 1916 & 4 & 3 & $35:$ & 42 & 1800 & 604 & $87430 !$ & 89834 & 450 & 17 & 2498 & 2139 \\
\hline
\end{tabular}

*) La mine de charbon d'Anju fut ouverte en 1914 avec cinq millions de yens de capital sous le régime spécial de la compagnie par actions en commandite (kabushiki gōshi gaisha 株式合資会社), elle est incluse dans les totaux à partir de cette date.

Source : CSTN, années 1914 et 1920 (t. 3).

33 Au-delà de ces données coloniales se pose néanmoins, à la vue des variations rapportées, la question de savoir sur quoi reposait cette croissance fulgurante des capitaux japonais eux-mêmes. Il s'agissait largement plus d'une conséquence de la spéculation boursière métropolitaine ${ }^{58}$ que d'un réel développement des forces productives en Corée. D'une part, nous retrouvons la rupture de 1916-17 évoquée à 
l'occasion du traitement de la question financière, d'autre part, nous constatons que la rupture fut le fait d'une percée massive du capital métropolitain en Corée, dans le contexte de surchauffe du «boum économique » au Japon évoqué en introduction.

Pour mieux comprendre ce changement, tentons d'en savoir plus à propos des activités sur lesquelles se concentraient les capitaux dont nous venons de parler.

\section{Les domaines d'activité et leur évolution}

Le tableau III montre la répartition du capital par secteur selon que le siège des entreprises était sur la péninsule ou en métropole.

Tabl. III : La capitalisation des entreprises actives en Corée en 1914

CPTL : CAPITAL (MILLERS DE YEN)

NBRE : NOMBRE D'ENTREPRISES

\begin{tabular}{|c|c|c|c|c|c|c|c|c|c|c|c|c|}
\hline & \multicolumn{4}{|c|}{$\begin{array}{l}\text { Siège en Corée } \\
\text { (pén in sulaires) }\end{array}$} & \multicolumn{3}{|c|}{$\begin{array}{c}\text { Siège au Japon } \\
\text { (métropolitaines) }\end{array}$} & \multicolumn{3}{|c|}{$\begin{array}{l}\text { entreprises } \\
\text { étrangères }\end{array}$} & \multicolumn{2}{|c|}{ TOTAL (b) } \\
\hline & cpt & nbr & /a & $/ \mathbf{b}$ & cptl & nbr & /a: / b & cpt & $\mathrm{Nbr}$ & $/ \mathbf{b}$ & cpt & /a \\
\hline Banques & 15005 & 23 & $39 \%$ & $37 \%$ & 25595 & 4 & $28 \%: 73 \%$ & - & .. & $\ldots$ & 40600 & $30 \%$ \\
\hline Commerce & 3822 & 88 & $10 \%$ & $11 \%$ & 27394 & 14 & $30 \%: 82 \%$ & 2080 & 3 & $36 \%$ & 33296 & $25 \%$ \\
\hline Transports & 2025 & 21 & $5 \%$ & $9 \%$ & 19500 & 2 & $21 \%: 91 \%$ & .. & .. & $\ldots$ & 21525 & $16 \%$ \\
\hline Gaz etélecari. & 827 & 11 & $2 \%$ & $13 \%$ & 5700 & 2 & $6 \%: 87 \%$ & .. & .. & 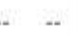 & 6527 & $5 \%$ \\
\hline Cie Tótaku & 10000 & 1 & $26 \%$ & $\begin{array}{l}100 \% \\
\end{array}$ & .. & -. & .. & -. & .. & .. & 10000 & $7 \%$ \\
\hline Pêche & 16 & 2 & $0 \%$ & $3 \%$ & 445 & 2 & $0,5 \%: 97 \%$ & .. & .. & .. & 461 & $0,3 \%$ \\
\hline Industrie & 2307 & 31 & $6 \%$ & $41 \%$ & 3348 & 6 & $4 \% ; 59 \%$ & .. & - & .. & 5655 & $4 \%$ \\
\hline Agriculture & 2145 & 18 & $6 \%$ & $37 \%$ & 3713 & 10 & $4 \%: 63 \%$ & .. & - & -. & 5858 & $4 \%$ \\
\hline Mines & 2175 & 3 & $6 \%$ & $29 \%$ & 5725 & 3 & $6 \%: 61 \%$ & 1493 & & $216 \%$ & 9393 & $7 \%$ \\
\hline тотАL (a) & 38591 & 209 & $\bar{i}^{2}$ & $9 \%)^{-7}$ & 91421 & 43 & $(68 \%)$ & 3573 & & $5(3 \%)$ & 133585 & $100 \%$ \\
\hline
\end{tabular}

*) électri. : électricité

N.B : il y a un maigre reliquat, néanmoins compris dans les totaux.

Source : CSTN, op. cit., année 1914.

Premier constat, en écho à notre précédente analyse: les entreprises coloniales métropolitaines étaient plus de deux fois mieux dotées en capitaux que les péninsulaires. Nous remarquons aussi la part écrasante des banques $(30 \%)$ et du commerce $(25 \%)$, puis celle des transports (16\%). Ensemble, ces trois secteurs représentaient près des trois quarts (71\%) des capitaux engagés. Ils constituaient le fer de lance d'une pénétration coloniale centrée sur le commerce : des banques pour financer les échanges, des entreprises de transport pour déplacer les marchandises et des compagnies marchandes pour gérer les transactions ${ }^{59}$. Les secteurs du commerce et du transport étaient à peu près exclusivement métropolitains (à 83 \%), signe de l'emprise coloniale, la capitalisation des banques péninsulaires et métropolitaines parait égale ( $48 \%$ contre $52 \%$ ). Mais les fonds propres des banques péninsulaires étaient eux aussi d'abord japonais, ce qui donc revient au même. Le domaine d'exercice privilégié de ces dernières était la vie économique intérieure de la péninsule : prêter aux paysans, aux marchands, aux propriétaires terriens et collecter leurs fonds. Dans un pays en pleine croissance démographique $(+24 \%$ d'habitants en dix ans, entre 1910 et 1920$)^{60}$, ces activités étaient prometteuses. En dehors de cette tête de classement, le secteur agricole se démarquait par son importance, occupant $11 \% \mathrm{du}$ total si nous y intégrons la Tōtaku. Ce secteur était très majoritairement péninsulaire (à $78 \%$, une évidence dans la mesure où la présence sur place des décideurs était une 
quasi-obligation dans ce type d'affaires. Les entreprises de gaz et électricité étaient certes importantes par le capital mobilisé et les innovations introduites (6,5 millions de yens), mais l'étaient peu par la portée matérielle de leur activité, limitée aux plus grandes villes. L'industrie de la pêche était le fait des pêcheurs japonais investissant les eaux coréennes qui leur étaient dorénavant pleinement ouvertes, mais avec $0,4 \% \mathrm{du}$ capital investi, l'impact économique en restait imperceptible. Les secteurs de la production industrielle constituaient un groupe de pointe technologique, mais très importants demandeurs de capitaux ils faisaient figure d'enfants pauvres de la colonisation à ses débuts. Comment cela a-t-il évolué ? L'examen du tableau IV nous montre l'état des choses en 1920.

Tabl. Iv : Les activités économiques du capital métropolitain en Corée en 1920

\begin{tabular}{|c|c|c|c|c|c|c|}
\hline & \multicolumn{6}{|c|}{ Capital métropolitain (milliers de yens) } \\
\hline & \multicolumn{2}{|c|}{ Sociétés par actions } & \multicolumn{4}{|c|}{ Total } \\
\hline & nominal & versé & nominal & vers & & $(1914)$ \\
\hline Commerce & 126800 & 118522 & 176995 & 168717 & $37 \%$ & $(27)$ \\
\hline Industrie & 87200 & 68275 & 87500 & 68575 & $15 \%$ & (3) \\
\hline Transport & 105000 & 65625 & 105500 & 66125 & $15 \%$ & (19) \\
\hline Banques & 66500 & 46025 & 66500 & 46025 & $10 \%$ & (25). \\
\hline Cie Tötaku & 51750 & 28587 & 21750 & 28587 & $6 \%$ & (10) \\
\hline Agriculture & 23130 & 15805 & 15134 & 12248 & $3 \%$ & (4) \\
\hline Mines & 35650 & 22840 & 36150 & 23340 & $5 \%$ & $(6)$ \\
\hline Pêche & 15130 & 12245 & 23222 & 15890 & $4 \%$ & $(0,4)$ \\
\hline Gaz, électricité & 18000 & 12300 & 18000 & 12300 & $3 \%$ & (6) \\
\hline TOTAL & 544160 & 399875 & 596751 & 452458 & $100 \%$ & $(100)$ \\
\hline
\end{tabular}

N.B. : les chiffres pour 1914 reprennent la colonne « siège au Japon » du tabl. IV en y incluant la Tōtaku.

Source : cSTN, année 1920, t. 3

37 Le commerce s'était nettement renforcé, dorénavant seul largement en tête (avec $37 \%$, il surpassait le total de ses deux dauphins). Les banques avaient perdu plus de la moitié de leur part (de $25 \%$ à $10 \%$ ) et les transports accusaient aussi une baisse (mais bien moindre, de $19 \%$ à $15 \%$ ). De même, le secteur agricole voyait sa part réduite de $14 \%$ à $9 \%$. Cela s'expliquait par le développement du secteur des manufactures et fabriques, certes encore faible ( $15 \%)$, mais très dynamique ( $3 \%$ seulement en 1914$)$, il se hissait désormais à la deuxième place (ex aequo avec les transports). L'emballement de la fin des années 1910 reposa donc sur le développement commercial et industriel. Autrement dit, comme on le voit, les statistiques construites par le gouvernement général de Corée dessinent assez clairement le schéma d'une colonisation économique "réussie», avec des débuts marqués par l'initiative politique, l'importance des entreprises publiques ou semi-publiques (banques et chemin de fer) et un développement assez rapidement marqué par l'importance du secteur privé, l'industrie et le commerce.

Se pose maintenant pour nous la question de savoir comment les autorités coloniales ont perçu ce secteur «industriel» durant les années 1910, alors même que 
l'historiographie a montré qu'il a surtout été développé durant la fin de la période coloniale, après 1930.

\section{Le secteur manufacturier et industriel}

Les statistiques coloniales utilisaient l'expression de kōjō 工場 (traduite traditionnellement par « usine ») pour désigner toute unité de production mue par une force mécanique ou employant plus de cinq ouvriers (shokkō 職工), dont la valeur de la production dépassait les cinq mille yens par an. Ainsi, les « usines" pouvaient aussi être des ateliers de taille moyenne. Or, si le nombre des unités de production industrielle et manufacturière a continuellement et fortement augmenté entre 1911 et 1917 (cf. tableau v), le capital investi en moyenne fondit, signe du développement de petites structures. Dans ce mouvement, une profonde différence s'est creusée entre unités coréennes et japonaises : si les premières étaient déjà en moyenne plus de cinq fois moins bien dotées en capital que les dernières en 1911, cet écart avait plus que triplé en 1919. Il fallait alors seize unités coréennes pour atteindre le capital moyen investi dans une unité japonaise. C'est là une évolution bien pire que celle esquissée dans notre analyse de la répartition générale des capitaux: six fois pires pour être exact (entre 1911 et 1920, l'écart des capitaux investis en moyenne dans les entreprises coréennes et japonaises n'avait crû que de moitié tandis que l'écart des capitaux investis dans les usines coréennes et japonaises tripla). Ainsi, le développement de la production industrielle en Corée a porté création de nombreuses petites structures, surtout coréennes, dont on peut peut-être penser qu'elles étaient au service des structures plus anciennes et plus importantes, très majoritairement japonaises ${ }^{61}$.

Tabl. v : La production manufacturière et industrielle en Corée coloniale (1911-1919)

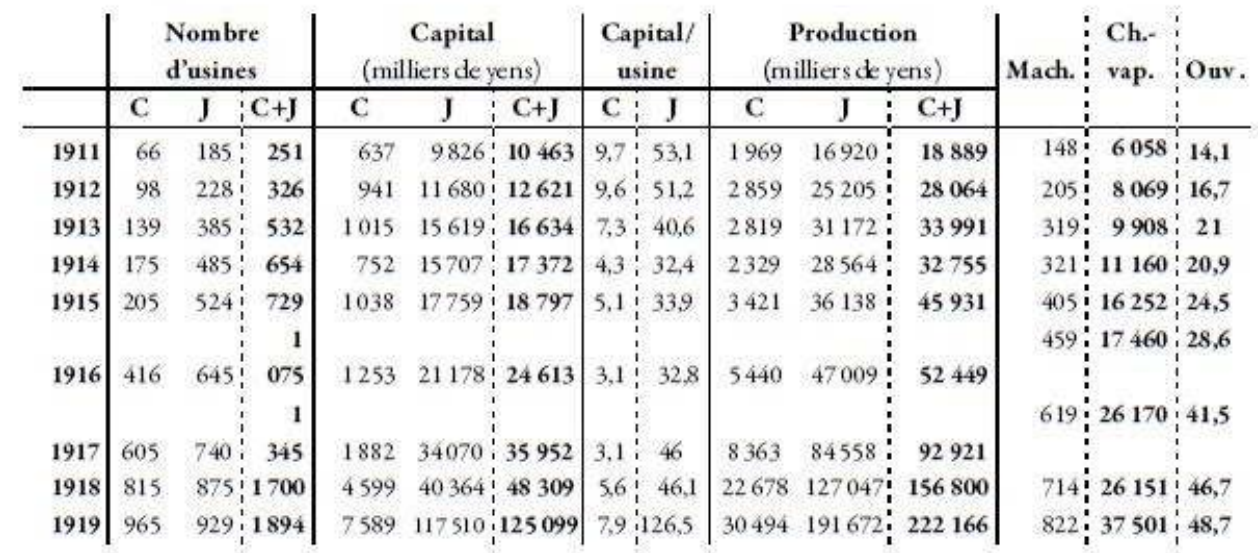

Mach. : nombre de machines ; Ch.-vap. : nombre de chevaux-vapeur (puissance mécanique).

Ouv. : nombre d'ouvriers, en milliers de personnes.

N. B. : entre 1917 et 1919, forte inflation au Japon.

Source : cSTN, chaque année.

Les unités japonaises écrasaient capitalistiquement des unités coréennes de plus en plus mal dotées, les premières s'arrogeaient manifestement les activités de pointe demandeuses de capitaux, les dernières restaient limitées aux activités les plus moins sophistiquées. Ainsi, la valeur de la production évolua elle aussi en faveur des entreprises japonaises : la leur fit plus que doubler $(+126 \%)$ tandis que la moyenne de 
leurs concurrentes coréennes stagnait. Compte tenu de l'inflation, il faut comprendre que la valeur moyenne réelle de la production des entreprises coréennes avait été divisée par deux tandis que celle des usines japonaises se maintint à un niveau stable. Or, en observant l'évolution du rapport entre la valeur de la production et le capital, nous voyons que l'écart entre industries coréenne et japonaise, après s'être d'abord creusé entre 1911 et 1915 (le secteur japonais à $+16 \%$, le coréen à $+7 \%$ ), se résorba en faveur de l'industrie coréenne en $1917(+24 \%$ contre $+35 \%)$ avant que la crise postguerre achevât de marquer le coup (le rapport du secteur coréen ne baissant entre 1917 et 1919 que de $10 \%$ tandis que celui du secteur japonais perdait un tiers de sa valeur). Manifestement, après que le "boum» de la guerre eut favorisé le développement et l'enrichissement de la production japonaise, la fin de la guerre signifia la fin de son envolée. Elle fut ainsi la première et la plus durement touchée, toutes proportions gardées. La crise n'effaça pas pour autant le gouffre colonial qui s'était creusé. Si le secteur coréen perdit moins que le secteur japonais, nous avons vu qu'il était surtout moins pourvu de capitaux... Il faudrait donc discuter des effets sociaux que cette "petite " perte provoqua. La bulle métropolitaine avait appauvri le tissu socio-économique coréen et son éclatement aggrava encore la situation. Au sein des entreprises japonaises, la crise postguerre impliqua surtout la concentration du capital (triplement de la moyenne du capital par entreprise entre 1917 et 1919) pour les entreprises concernées.

41 En termes d'activités, malgré le qualificatif « industriel » que nous utilisons, la réalité de la production restait celle d'une économie d'abord agricole. Selon les annuaires statistiques coloniaux, plus de la moitié de la production « industrielle » $(57 \%)^{62}$ était le fait des raffineries de riz, un secteur plus crucial encore pour les Coréens ( $72 \%$ de leur production) que pour les Japonais ( $55 \%$ ). Cette domination a faibli pendant la période, baissant de $63 \%$ en 1911 jusqu'à $49 \%$ en 1916, avant de remonter aussitôt à $56 \%$ sur 1918-19. Cette remontée s'explique notamment par la forte croissance des exportations de riz vers la métropole (voir ci-après) dans ces années, un fait qui explique aussi que "l'industrie » coréenne ait mieux résisté (en termes de dynamique) à la crise que le secteur japonais. En dehors de ce domaine clé, le travail des métaux et la fabrication du tabac étaient deuxièmes tous les deux ( $13 \%$ chacun sur la période). Si le secteur du tabac a conservé une part stable malgré une mauvaise passe entre 1916 et 1917 (à 7 \%), le parcours de la métallurgie a été bien plus chaotique : après avoir cru depuis un petit $4 \%$ en 1911 jusqu'à $27 \%$ en 1917, il revint à un niveau insignifiant $(6 \%)$ en 1919 (on reviendra sur cette crise). Filatures textiles, tanneries, brasseries, ateliers de céramiques, etc. composaient les $20 \%$ restants de la production. Un rapport officiel publié en 1922 sur « les progrès » effectués en Corée entre 1918 et 1921 ne pouvait pas cacher la réalité de la crise des secteurs non agricoles : il ne s'étendait pas en détail sur celle-ci, mais l'évoquait néanmoins à plusieurs reprises et insistait tactiquement, pour expliquer la crise, sur les conditions " anormales » du développement dû aux effets de la guerre en Europe ${ }^{63}$. Un constat contrastant avec les éloges adressés au secteur agricole, dorénavant destiné à fournir à grande échelle et de manière systématique un riz bon marché à la métropole.

Enfin, pour comprendre la véritable dimension de ce "développement industriel », il faut considérer sa puissance mécanique et le nombre d'employés : 48705 ouvriers pour 1900 sites de production en 1920, mais seulement 828 machines mues par 37501 chevaux-vapeur ${ }^{64}$. Il y avait plus d'ouvriers que de chevaux-vapeur, la limite du 
développement « industriel » en Corée en est bien résumée. La part japonaise dans ce mouvement était importante, employant 22747 personnes en 1916, dont 18279 Coréens $^{65}$. Dans un pays peuplé de quinze à seize millions d'habitants, cela restait malgré tout peu de chose... comme les infrastructures et la colonisation agricole, la production industrielle connut un développement limité et des difficultés importantes en fin de période.

Voyons maintenant ce qui relève du secteur commercial, théoriquement le plus important de tous, autour de deux cas précis : le riz et le fer.

\section{La capture commerciale du riz et du fer}

44 Comme on l'a vu précédemment, le "commerce » coréen était en réalité largement captif de sa relation avec le Japon, et l'on ne peut donc discuter sans un recul prudent les chiffres de la «balance commerciale » proposés par le gouvernement général. (Une analyse de ces chiffres nécessiterait par ailleurs une étude serrée des "prix » pratiqués dans ces «transactions»). Dans ce contexte de domination économique, l'article d'exportation le plus vendu à la métropole, le riz ( $38 \%$ de la valeur des exportations totales vers le Japon) revêtait une importance particulière. Une autre denrée comptait beaucoup dans la relation économique avec la métropole, bien que ne représentant que $1 \%$ de la valeur des exportations coréennes vers le Japon sur la période : le minerai de fer, indispensable au développement industriel sur l'archipel.

\section{La question du riz}

À partir de la fin des années 1900, alors que la formation de l'empire colonial achevait une première étape, "la question du riz " s'imposait peu à peu, mais assurément en termes de crise au Japon. La production métropolitaine stagnait sous les cinquante millions de koku tandis que la population croissait fortement ( $+11,8 \% /$ an en moyenne entre 1900 et 1920, de 43,8 millions à 56 millions d'hab.). Sur la décennie 1900, quatre millions de koku manquèrent chaque année ${ }^{66}$. À cela s'ajoutait un schéma particulier du marché international du riz au Japon: les marchands exportaient le meilleur riz japonais, réputé dans le monde, et importaient à bon marché du riz étranger de qualité inférieure ${ }^{67}$. Ils tiraient ainsi profit des deux extrémités du marché. Cet état des choses faisait monter le plafond des prix des riz les plus chers et abaisser le plancher de la qualité alimentaire des riz les moins chers, consommés par les plus pauvres. Ce schéma avait commencé de se mettre en place au cours des années 1890 et avait déjà suscité quelque agitation localement, et très ponctuellement. La hausse des cours du riz $(+30 \%$ entre 1890 et 1910) ${ }^{68}$ satisfaisait les grands propriétaires terriens, mais suscitait un croissant mécontentement populaire, favorisant les conflits sociaux et menaçant d'aboutir à une augmentation forcée des salaires dans l'industrie. Le développement industriel en métropole était le cœur du problème : plus de travailleurs appelés dans l'industrie, c'était autant de bras enlevés à la production agricole. Ces ouvriers toujours plus nombreux, il fallait continuer de les payer moins, et pour cela, importer plus de riz coréen bon marché était une solution. 


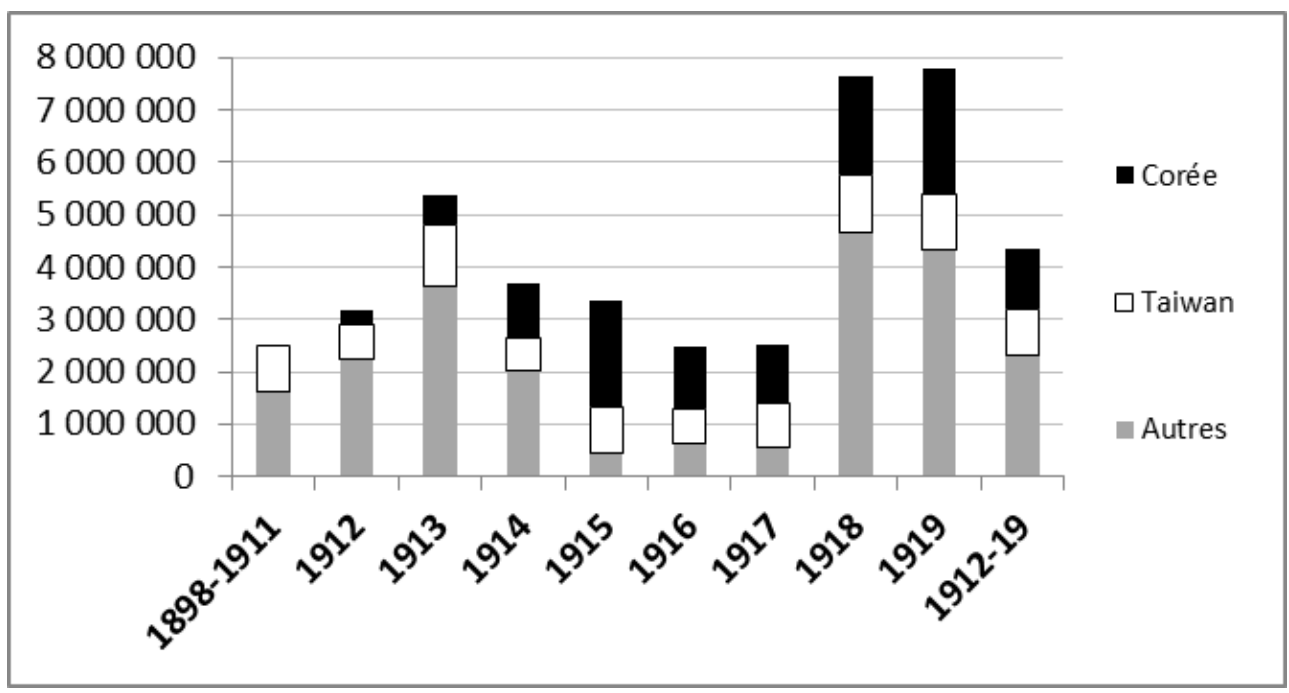

Source : Nōshōmu-shō nōmu-kyoku, Kome no tōkei tekiyō, op. cit., 1920.

Dans un premier temps, l'importation de riz étranger bon marché s'en trouva favorisée (voir graph. x) : le Japon importait encore à la fin des années 1900 plus d'un demi-million de $k o k u^{69}$ de riz indochinois ${ }^{70}$. Dans les années 1900 et 1910 , cette situation gagna progressivement en importance, s'installant dans la durée tout en restant limitée aux basses couches sociales. Loin des ors du gouvernement, le problème se développait et par à-coups, comme en 1911, et se faisait plus pressant. Cette année-là, une mauvaise récolte en métropole causa un doublement des importations de riz (d'un peu plus d'un million à plus de deux millions de $k o k u)^{71}$. Face à un aléa climatique, les masses restaient silencieuses, encore peu conscientes de la lente et fatale évolution en cours, mais les pouvoirs économiques, y compris le colonat français d'Indochine, s'intéressaient de près à cette « question du riz $»^{72}$. En Indochine, on guettait une baisse des tarifs douaniers japonais pour pouvoir pénétrer le marché à grande échelle (le riz représentait alors près des deux tiers des exportations indochinoises). C'était en vain, le pouvoir politique japonais restait sous la puissante influence des grands propriétaires terriens ${ }^{73}$. Ce n'est qu'au terme de notre période, face aux émeutes de 1918 que Tōkyō abolit provisoirement la taxe à l'importation (entre le $1^{\text {er }}$ novembre 1918 et le 31 octobre 1919). Jusque-là, plutôt que de payer des importations en monnaie-or, la métropole trouva dans les achats de riz coloniaux une double solution : développer le régime économique colonial et nourrir à bon marché les classes laborieuses toujours plus nombreuses au Japon, évitant la hausse des salaires. Première colonie japonaise, Taiwan fut d'abord mise à contribution : moins peuplée et dotée d'un climat subtropical propice à la riziculture, l'̂lle fournit près d'un quart (23\%) des apports d'outre-mer au Japon jusqu'en $1918^{74}$, exportant près d'un cinquième (18 \%) de sa production ${ }^{75}$. Cette contribution très importante au regard de l'étroitesse du territoire et de son faible peuplement était son maximum. Les capacités productives de Taiwan étaient limitées à un surplus d'un peu moins d'un million de koku annuels entre 1905 et $1920^{76}$, et surtout, la culture du riz ne devait pas pouvoir concurrencer celle du sucre. Ce dernier phénomène, le «face-à-face du riz et du sucre » (beitō sōkoku 米糖相兛), sera devenu crucial dans les années 1920, mais était déjà sensible sur notre 
période. Dans ce contexte, les achats de riz coréen par la métropole allèrent croissants (graphique $\mathrm{x}$ ).

Importer du riz coréen a d'abord été difficile: la production locale n'était pas excédentaire, la population coréenne était elle-même en forte croissance démographique (de 13,3 millions en 1910 à 17,3 millions d'hab. en 1920) et le développement de la riziculture n'a pas été aussi fort que souhaité. D'autre part, le gouvernement japonais n'a pu détaxer les importations qu'après l'annexion du territoire, en avril 1912, alors même qu'il s'était engagé auprès des puissances à ne pas modifier le régime douanier coréen avant $1916^{77}$. Cette mesure qui montre bien la dimension pressante du problème. Malgré cela, sur un marché japonais d'importations sans pic notable entre 1913 et 1918, le riz coréen s'imposa face aux riz indochinois, birman et chinois (graph. x). Cela, à la faveur de la guerre ${ }^{78}$ : les colonies européennes ne trouvaient plus de navires marchands disponibles pour le Japon, la guerre mobilisant ces derniers sur l'espace européen. Ainsi, entre 1914 et 1917, les riz coloniaux japonais représentaient $80 \%$ du volume importé. La métropole accrut ainsi sa déjà très importante part dans les exportations de riz coréen (de $81 \%$ entre 1912 et 1916 à $90 \%$ entre 1917 et 1921) ${ }^{79}$. En 1918, ces achats métropolitains massifs créèrent pour la première fois un surplus commercial en faveur des grands propriétaires coloniaux de Corée.

Comparant les données des périodes 1898-1911 et 1912-19 (graph. X), nous constatons l'importance de l'apport coréen: il satisfit $62 \%$ de la croissance des importations, réduisant l'appel aux riz étrangers à $37 \%$ de celle-ci. Si notre graphique fait apparaître les années de guerre comme un creux, la ponction japonaise en Corée pesa surtout à partir de ce moment : la valeur du riz coréen exporté en métropole ne représentait que $4 \%$ de la production entre 1910 et 1913, mais déjà le double en 1914 et jusqu'à $18 \%$ et $21 \%$ en 1915 et 1919 (pour une moyenne de 13 \% sur 1914-1919). Peu importaient les conditions de l'offre coréenne, malgré les aléas des récoltes et la croissance démographique, la demande japonaise était souveraine, de plus en plus écrasante. N'est-ce pas pour échapper à cette capture coloniale que la production coréenne de blé, soja et millet italien doubla quasiment entre 1910 et $1920^{80}$ ? Dans le graphique II, la force de ce tournant est lisible dans la diminution croissante de la part du riz dans le volume des produits agricoles transportés. Le boum démographique de ces années explique que les cultivateurs coréens aient cherché à produire des denrées d'abord pour eux et le marché environnant, plutôt que du riz en terrains inondés pour la métropole. Pour ce dernier, non seulement un investissement plus lourd était nécessaire, mais surtout la production était soumise aux pressions aléatoires d'un lointain marché aux intermédiaires nombreux et puissants. Face à l'instabilité du commerce au loin et à la donne particulièrement inégale du marché colonial, la production locale se réfugia vers des niches intérieures, les trouvant dans une économie locale restée principalement vivrière ${ }^{81}$. Ce n'est que dans les années 1920 que le secteur rizicole coréen sera modernisé, via un programme sur quinze ans mené par le gouvernement général, tandis que la taxe à l'importation vers la métropole était abolie en 1919. Comme noté, le Japon métropolitain accusait le coup des «émeutes du riz » de l'été 1918, et accusait l'échec de la politique commerciale de la décennie passée en Corée : il allait encore raffermir son emprise sur l'agriculture coréenne.

Nous avons noté que cette "question du riz " coréen allait de pair avec la question industrielle: fournir un riz bon marché pour maintenir les salaires du prolétariat 
japonais, c'est-à-dire les coûts de production, au plus bas était crucial pour l'industrie japonaise et son développement. Pour cette question industrielle, la colonie coréenne revêtait une importance aussi pour l'approvisionnement en minerai de fer.

\section{Le minerai de fer coréen}

50 Le développement de la sidérurgie au Japon était contemporain de la constitution de l'empire colonial : entre 1897 et 1901, le premier complexe sidérurgique moderne était construit et inauguré à Yahata ${ }^{82}$, dans le nord de Kyūshū, à proximité du bassin houiller du Chikuhō et non loin des mines de fer coréennes et chinoises ${ }^{83}$. Nous intéressent ici les questions de l'approvisionnement en minerai de ces complexes sidérurgiques, et de la place du minerai coréen au sein de cette configuration.

51 Car le Japon restait dépendant de ses imports d'acier et fonte étrangers, et, afin de pallier ces achats et produire lui-même, il devait importer massivement le minerai de fer et les charbons spéciaux nécessaires au travail sidérurgique. Dans ce contexte, son implantation en Corée lui fournit de précieuses ressources.

52 Quantitativement et qualitativement, le minerai de fer japonais ne pouvait satisfaire la demande sidérurgique nationale. La plus proche source d'approvisionnement extérieur était la Corée, sur laquelle la colonisation de la péninsule permit un contrôle total. En 1917, les principales mines (à Unryul et Chaeyŏng) étaient tenues par Tōkyō mais les zaibatsu japonais étaient eux aussi actifs : Mitsui à Kaechon, Mitsubishi à Hwangju et Chaeyŏng, ainsi que le capital minier du Chikuhō (familles Yasukawa et Asō) ${ }^{84}$. Toutefois, la production coréenne ne suffisait pas et les gisements chinois de Daye (en Chine centrale, actuelle région de Wuhan) furent sollicités (cf. graph. XI). Par leur qualité ces derniers étaient les meilleurs à disposition et le capital japonais s'en assura un approvisionnement régulier par des prêts accordés au consortium gérant la mine dès $1903^{85}$. Le graphique XI montre comment les importations de Corée, bien qu'importantes ( $26 \%$ du total entre 1912 et 1920), ont toujours été devancées par l'apport chinois ( $46 \%$ du total). On constate aussi la "bulle » de l'immédiat aprèsguerre dans la hausse des imports (de 1918 à 1920) et son dégonflement en 1921-22 (grave crise financière en 1922). Cette chute fut particulièrement dure pour le minerai coréen, qui était alors supplanté par le minerai des "Colonies du Détroit» (les possessions britanniques sur le détroit de Malacca), Johore en l'occurrence ${ }^{86}$. Cette bulle et son effondrement concernaient directement tout le secteur sidérurgique japonais d'alors. 
Graph. XI : Demande et importations du minerai de fer au Japon (1912-1922) (unité : tonnes)

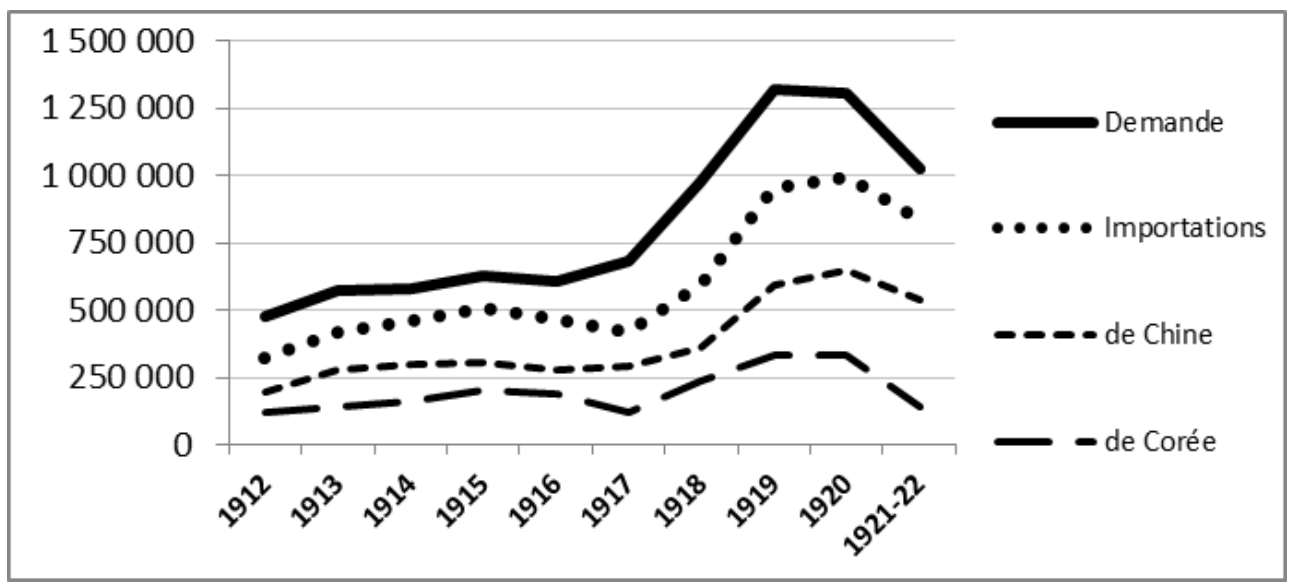

NB : la demande correspond ici à la production ajoutée aux imports, exports retranchés. Source: Shōkō-shō kōzan-kyoku 商工省鉱山局 (Bureau des Mines du ministère du Commerce et l'Industrie), Seitetsugyō sankō shiryō 製鉄業参考資料 (Documentation sur I'Industrie sidérurgique), enquête de juin 1926, p. 6.

\section{La sidérurgie japonaise en Asie du Nord-Est}

Après avoir pensé que la guerre ne durerait pas, face à la « famine du fer » (tetsu no kikin 鉄の飢䬰 $)^{87}$ et au quasi-doublement du cours de la fonte sur la seule année 1915, le capital japonais se lança à partir de 1916 dans la construction d'aciéries vouées à remplacer les imports faisant défaut. Cette «famine» était particulièrement grave : sans fer ni acier en quantité suffisante, le Japon ne pouvait pas jouir à plein de l'effet d'aubaine de la Grande Guerre puisqu'il lui fallait armer des navires pour soutenir son expansion commerciale et assurer le développement d'une industrie lourde capable de fabriquer les machines et l'outillage toujours nécessaires à son industrie légère. Privé des sources européennes, le Japon se trouva à partir de 1916 dépendant d'une offre américaine peu dispendieuse (cf. graph. XII). L'entrée des États-Unis dans la guerre en avril 1917 fit aussitôt peser la perspective d'un embargo total sur l'acier, le problème devint une crise aiguë. L'embargo fut effectif à partir d'octobre, mais entretemps les commandes s'étant multipliées et les cours avaient déjà flambés8. Au-delà d'octobre, Tōkyō n'obtint des livraisons qu'à la condition que l'acier fût utilisé pour armer des navires destinés à servir l'effort de guerre sur l'océan Atlantique ${ }^{89}$. Ce faisant, les importations purent être maintenues, mais à des prix très élevés ${ }^{90}$. 


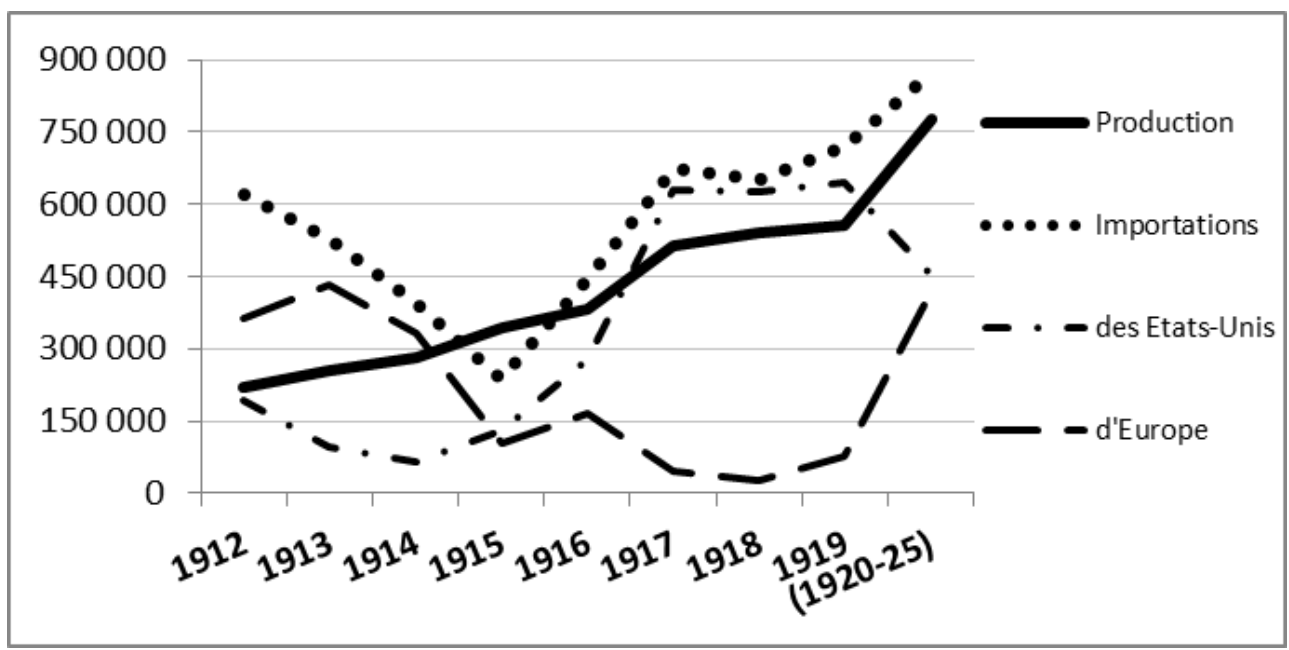

Source : Shōkō-shō kōzan-kyoku, Seitetsu-gyō sankō shiryō, op. cit.

Cette crise suscita au Japon un « boum de la fièvre sidérurgique » (seitetsu-netsu no bokkō 製鉄熱の勃興 $)^{91}$ entre 1917 et 1918 . Les plus importants projets d'aciéries se sont concentrés à proximité des gisements de fer en Corée et Mandchourie méridionale, comme le conglomérat ōkura à Benxihu 本溪湖 (1915) et la CSM à Anshan 安山 (1918). Mitsubishi bâtit un complexe autour de sa mine de fer coréenne à Kyumip'o (entre P'yŏngyang et Chinnamp'o), produisant du sulfate d'ammoniaque à partir de 1917, de la fonte à partir de 1918 et de l'acier à partir de $1919^{92}$. Sa production était destinée à répondre aux besoins de l'industrie navale dans laquelle excellait le groupe Mitsubishi et les premières commandes furent enregistrées dès août $1917^{93}$. Ces projets japonais furent pour la plupart concrétisés en 1918, année de la fin de la guerre en Europe, exactement au moment où les cours mondiaux de l'acier s'effondraient. La valeur de la production de fonte coréenne chuta ainsi de près de moitié entre 1918 et 1920 (de 15,37 à 8,27 millions de yens) ${ }^{94}$, un début catastrophique.

Les investissements effectués au plus fort de la bulle par le capital japonais durent affronter le retour de la concurrence occidentale, et ce non seulement sur la sidérurgie, mais dans tous les secteurs commerciaux que les économies occidentales avaient délaissés pendant les années de guerre. Deux ans à peine après avoir titré sur son «boum» et sa "fièvre», la presse nationale criait au "naufrage du secteur sidérurgique " (seitetsu-jigyō no botsuraku 製鉄事業の 没落 $)^{95}$. Les spéculateurs se détournèrent aussitôt du secteur pour parasiter la bourse et les commerces classiques comme celui du riz. C'était la généralisation de la bulle ${ }^{96}$.

\section{L'économie coloniale de Corée à la fin de la Première Guerre mondiale : de la spéculation à l'impasse}


Graph. XIII : Évolution indiciaire des prix de produits et denrées alimentaires et industriels au Japon et en Corée (1914-1919)

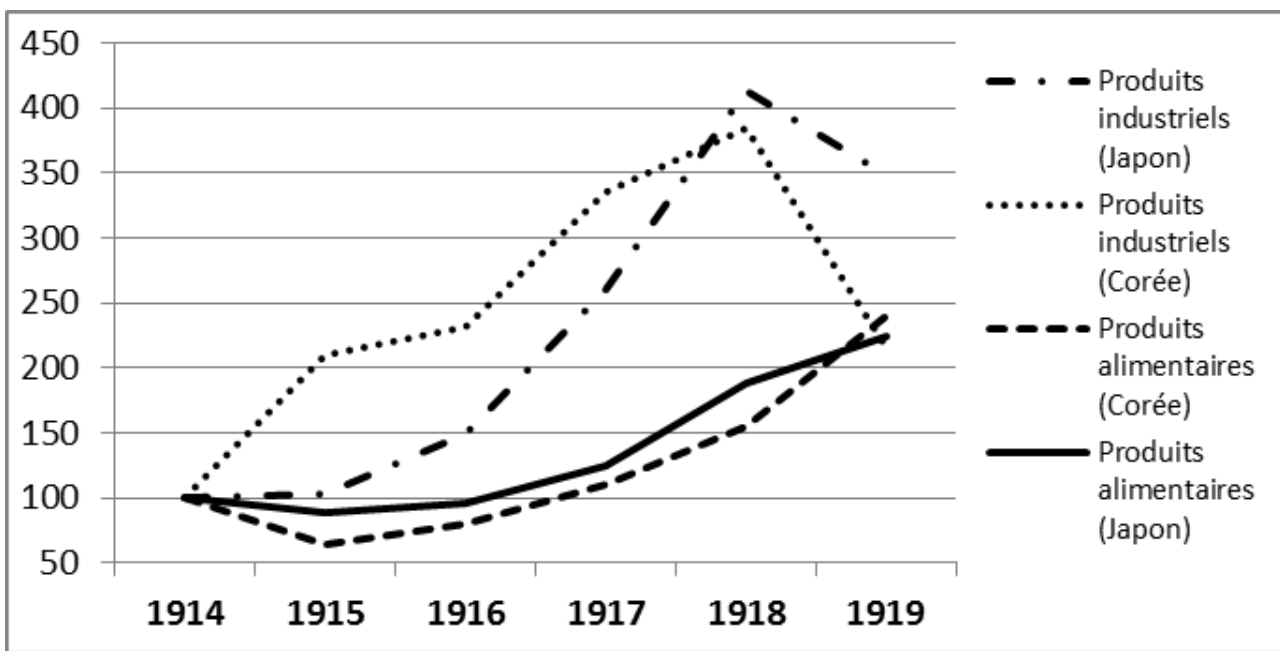

Source : Pour le Japon, produits industriels (acier importé, fonte, charbon, pétrole et cotonnades) et agricoles (riz, soja, blé) : United States Tariff Commission, The Foreign Trade of Japan. A Study of the Trade of Japan with Special Reference to That with United States, Washington, Government Printing Office, 1922, 229 p., p. 22 ; sauf pour l'acier importé : Shōkō-shō kōzan-kyoku, Seitetsu-gyō sankō shiryō, op. cit.

Pour la Corée, produits agricoles : cSTn, chaque année (moyenne des prix des riz décortiqués et raffiné et du soja) ; produits industriels (miniers, métaux et textiles) : kim Nak Nyeon, Nihon-teikokushugi-ka no chōsen keizai, op. cit., p. 181.

La montée des cours des produits industriels avait déjà tiré à la hausse ceux des denrées alimentaires (graph. XIII), mais lorsque les premiers chutèrent en 1919 alors que les seconds continuaient d'augmenter, la bulle industrielle devint un grave problème social, tout particulièrement en Corée où les achats massifs de la métropole exportaient la montée des prix. Le graphique XIII montre à quel point la colonie subit les tendances de la métropole: les cours coréens des produits agricoles devaient être inférieurs et ceux des produits industriels supérieurs aux cours métropolitains, jusqu'à la surchauffe du système en 1918 et son explosion en 1919. Situation paradoxale en Corée : on eut à payer moins cher les produits industriels seulement lorsque la production coréenne elle-même s'écroula, exactement au moment où le prix des denrées alimentaires augmentait.

Pour le régime colonial, le problème de la hausse du coût de la vie était crucial : il était identifié par les enquêtes de police comme l'une des principaux griefs adressés par la population au nouveau régime dès les années 1911-1914.7, avant même que le mouvement des prix ne devînt critique. Le 28 février 1919, le Keijō nippō, journal local surtout lu par les résidents japonais, achevait une série de cinq articles dénonçant un pétrole devenu " plus cher que l'alcool de riz » et un charbon de bois « plus cher encore que tout bien comestible $»^{98}$. La politique menée en Corée entre 1905 et 1918-1919 était une impasse économique et sociale. Le lendemain, deux jours avant que l'ex-roi coréen Kojong ne fût inhumé, un peu plus d'un an après le discours du président américain Wilson sur le "droit des peuples à disposer d'eux-mêmes » et dans le contexte d'une Europe elle-même marquée par les révolutions socialistes ou l'instabilité sociale (Russie, Allemagne), un groupe d'intellectuels militants coréens se réunit dans la capitale coréenne pour déclarer en public l'indépendance de la Nation et appeler au soulèvement. Ce fut comme une étincelle sur une plaine d'herbes sèches : la société tout 
entière réagit, le «Mouvement du $1^{\mathrm{er}}$ mars » était lancé, ébranlant la domination coloniale.

Tant dans le domaine des infrastructures que dans celui des structures de production ou le commerce, la première période du régime colonial que nous avons vue dans cet article n'a pas permis le développement d'une économie stable dans la péninsule.

Jusqu'en 1916 les investissements restèrent insuffisants et lorsqu'à partir de 1916 ils devinrent importants, ce fut dans le cadre de la bulle spéculative de la Grande Guerre, voire en relation avec la Mandchourie. Par ailleurs, quoi qu'en disent les annuaires statistiques du gouvernement général, qui insistent lourdement sur le "coût de la colonisation » comme on l'a vu, l'économie de la péninsule restait et restera largement écrasée par des ponctions métropolitaines permanentes sur les ressources naturelles et par un jeu du capital introduit tout à la faveur de la propriété japonaise. La Corée coloniale était tel un tonneau percé en réalité siphonné par ce qui n'était pas tant un déficit commercial et public que plutôt un vase communicant en faveur de la métropole.

60 Se pose donc la question du regard et du discours portés par la documentation utilisée ici. On voit que le gouvernement général aime à se présenter comme si la Corée était un État indépendant, jouissant de réelles prérogatives commerciales, et comme s'il existait encore, après 1910, un «État » en Corée dont l'on pourrait analyser le déficit public et la balance commerciale. Mais ces notions ont-elles un sens en contexte colonial? En effet, que représentent des "prêts" accordés par la métropole au gouvernement général de Corée, en réalité hypothéqués sur la richesse coréenne et servant à raffermir l'appareil colonial japonais? Celui-ci, évidemment, ne «remboursait» jamais la métropole, si ce n'est les investisseurs : il s'agissait d'un jeu bien compris dans lequel les valeurs monétaires et financières ne circulaient que dans un seul sens. De même, la question du rôle de la Banque coloniale de Corée qui, comme la Banque d'Indochine dans le cas français, servit surtout de tremplin régional aux investisseurs de métropole, se pose quand l'on veut analyser le secteur financier de la Corée coloniale.

61 Au final, les informations officielles sur l'économie de Corée produites par le régime colonial ne montrent-elles que le développement de... l'appareil japonais en Corée colonisée, sans que l'on puisse saisir précisément le tissu économique coréen concret (sauf dans ses liens avec les réseaux économiques japonais, plus ou moins marqués selon les secteurs). Il faudrait par ailleurs analyser la construction statistique de la «comptabilité » du gouvernement général: nous avons montré, en effet, que les chiffres produits par ce dernier avaient parfois été infirmés par des historiens de l'économie.

62 Avec la fin de la guerre, en 1918, l'échec des premiers projets d'industrie lourde et la brutale hausse du coût de la vie entre 1916 et 1919 en Corée, marquèrent le coup. C'est dans ce contexte que des auteurs japonais critiques comme Yoshino Sakuzō 吉野作造 (1878-1933) ou Ishibashi Tanzan 石橋湛山 (1884-1973) dénoncèrent le fardeau croissant et inutile de l'empire colonial ${ }^{99}$. Ishibashi Tanzan, notamment, expliqua que le Japon gagnerait à se développer de façon repliée sur l'archipel, loin des " chimères » de la présence armée en Asie orientale ${ }^{100}$. Il n'empêche: l'effort développé dans les années 1920 afin de moderniser l'agriculture de la péninsule, puis celui effectué dans l'industrie lourde à partir des années 1930 allaient pourtant donner un autre poids à l'économie coloniale de Corée, tout en raffermissant sa position vis-à-vis du Japon et vis-à-vis de l'Asie du Nord-Est. En ce sens précis, l'échec de la politique de 
développement des années 1910, puis le Mouvement du $1^{\mathrm{er}}$ Mars 1919, amenèrent à une autre politique économique dans la colonie qui allait autrement complexifier la structure de la Corée coloniale, ainsi que les rapports entre colonisé et colonisateur.

\section{NOTES}

1. Celui-ci déclarait en 1901 dans L'Impérialisme, spectre du vingtième siècle: "Même si l'établissement des empires ne peut qu'accélérer la voie vers la chute et la ruine, les pays occidentaux ont la capacité de maintenir leur drapeau pendant quelques années et de s'en enorgueillir. Le Japon serait, lui, incapable de tenir un seul jour l'empire qu'il viendrait à construire. » Traduit par Christine Lévy, CNRS éditions, 2008, 188 p., p.177. Sur Kōtoku voir l'introduction détaillée de Christine Lévy à sa traduction de l'ouvrage précité (p. 1-79).

2. En langue occidentale on parlait alors de «Formose» (Formosa), taiwan étant la lecture (tant chinoise que japonaise) des caractères 台湾.

3. Péninsule de $55000 \mathrm{~km}^{2}$ constituant la majeure partie du littoral mandchourien méridional.

4. Au sujet de la question précoloniale de la Corée au Japon, nous renvoyons à Lionel Babicz, Le Japon face à la Corée à l'ère Meiji, Maisonneuve et Larose, 2002, 271 p. Et de manière plus générale, sur la Corée coloniale (jusqu'en 1945), nous renvoyons à Alain Delissen, « La péninsule coréenne aux $\mathrm{XIX}^{\mathrm{e}}$ et $\mathrm{XX}^{\mathrm{e}}$ siècles ", dans Hartmund Rotermund et alii, L'Asie orientale et méridionale aux XIX $X^{e}$ et XXe siècles, PUF, « Nouvelle Clio », 1999, CCXLIV + 546 p., p. 177-195.

5. Treize millions selon les statistiques coloniales, mais un peu plus de seize millions selon la reconstitution de Myung Soo Cha 車明洙, « Keizai seichō shotoku bunpai kōzō henka » 経济成 ·

長所得分 - 配構造変化 (Croissance économique, répartition des revenus et changements structurels), dans Kim Nak Nyeon (dir.), Shokuminchi-ki chōsen no kokumin keizai keisan 植民地期朝 鮮の国民経済計算 (Une quantification de l'économie nationale coréenne pendant la période coloniale), Tōkyō daigaku shuppan 東京大学出版, 2008, 640 p., p. 322-329.

6. 540 millions de yens d'exportations contre 399 d'importations entre 1915 et 1918.

7. Cette tendance se retrouve de manière générale dans les travaux portant sur les colonies (histoire culturelle, politique, etc.). Voir : Nihon shokuminchi kenkyūkai 日本植民地研究会 (éd.), Nihon shokuminchi kenkyū no genjō to kadai 日本植民地研究の現状と課題, Atena-sha アテナ社, 2008, 254 p., notamment le chap. 3 portant sur la Corée (Mitsui Takashi 三ツ井崇, «Chōsen » (Corée), p. 92-119). Pour un compte-rendu actuel (mais ignorant les travaux en langues occidentales) voir matsumoto Takenori 松本武祝, « Keizai-shi » 経済史 (L'histoire économique), dans chōsen-shi kenkyū-kai 朝鮮史研究会 (dir.), Chōsen-shi kenkyū nyūmon 朝鮮史研究入門 (Une introduction à la recherche en Histoire de la Corée), Nagoya daigaku shuppan 名古屋大学出版 2011, 530 p., p. 256-266.

8. Elle se retrouve notamment dans les travaux de l'école économétrique (Mizoguchi Toshiyuki 溝口敏行, Yamamoto Yūzō 山本有造 et Kimura Mitsuhiko 木村光彦 pour citer ceux auxquels nous nous référerons ici). Les travaux américains relèvent en général de cette catégorie (mais pas tous, comme la controverse Bix-Myers l'a montré : Ramon H. Myers et Herbert Bix, "Economic Development in Manchuria under Japanese Imperialism: A Dissenting View", The China Quarterly, no. 55, juil.-sept. 1973, p. 547-559).

9. Comme Samuel Ho dans "Colonialism and Development: Korea, Taiwan, and Kwantung" (in Ramon H. Myers et Mark R. Peattie (dir.), The Japanese Colonial Empire.1895-1945, Princeton 
University Press, 1984, 540 p.) disant en introduction que « le développement colonial a amélioré les conditions de vie des populations colonisées » (p.347) et répétant en conclusion que les bénéfices/bienfaits (benefits) de la colonisation ont été « largement partagés » avec les colonisés, avant de déclarer aussitôt que la force coloniale japonaise a «bien capturé une large part des gains » (p. 386).

10. Auteur d'un ouvrage-référence sur la question agraire coloniale... mais focalisant sur les années 1920 et 1930, la période des années 1910 ne bénéficie d'aucune analyse particulière (l'analyse du cas de la Chōsen kōgyō kaisha, p. 146-166, utilise des données couvrant aussi entièrement les années 1910, mais le corps de texte ne s'attarde pas dessus). Asada Kyōji 浅田喬 二, Nihon teikokushugi to kyū-shokuminchi jinushi-sei 日本帝国主義と旧植民地地主制 (L'impérialisme japonais et le régime des propriétaires terriens dans les anciennes colonies), Ryūkei shosha 龍渓書舎, 1989, 343 p. (éd. augmentée de la première éd. en 1968), p. 67-166 (chap. 3).

11. Kaneko a publié dans les revues scientifiques une série de travaux sur les investissements japonais en Mandchourie. Kaneko Fumio 金子文夫, Kindai nihon niokeru tai-manshū tōshi no kenkyū 近代日本における対満州投資の研究 (Une étude des investissements japonais en Mandchourie), Kondō shuppan-sha 近藤出版社, 1990, 546 p.

12. Pour rapporter une critique de l'école "positive» à leur égard: Ramon Myers déclara "paradoxal que les historiens japonais [centrés sur le thème de l'exploitation] admettent qu'une révolution agricole a eu lieu en colonies, comme en Corée, tout en niant que cette révolution eût des retombées positives [favorable benefits] pour les peuples colonisés " (R.H. Myers, "Post-World War II Japanese historiography of Japan's Formal Colonial Empire", in Ramon H. Myers et Mark R. Peattie (dir.), The Japanese Colonial Empire. 1895-1945, op. cit., p. 469). Myers n'appuie cette assertion d'aucune référence renvoyant à des historiens marxistes japonais ayant reconnu une « révolution agricole » coloniale.

13. L'historiographie sud-coréenne s'est d'abord construite contre "la thèse de la stagnation » (teitai-ron 停滞論 en japonais) de l'économie précoloniale - commune à tous les colonialismes. Voir Alain Delissen, op. cit., p. 143 et 156, ainsi qu'Iwamoto Takunari cité plus bas en note 15.

14. À tel point que la série des «Leçons Iwanami » consacrée à l'histoire du fait colonial intitula son volume économique « Industrialisation et colonisation » (ōe Shinobu 大江志乃夫 et alii (dir.), Kindai nihon to shokuminchi 近代日本と植民地 (Le Japon moderne et les colonies), vol.3, «Shokuminchika to sangyōka » 植民地化と産業化 (Colonisation et industrialisation), Iwanami shoten 岩波書店, 1993, 307 p.). Voir par exemple l'introduction de la contribution de Kaneko Fumio («Shokuminchi-tōshi to kōgyōka » 植民地と工業化 (Les investissements en colonies et l'industrialisation), dans ibid., p. 27-28). Cela se retrouve aussi, mais avec nuance, dans PierreFrançois souyri, «Le colonialisme japonais », dans Marc Ferro (dir.), Le livre noir du colonialisme, Hachette, 2004, 1124 p. (première édition : Paris, Laffont, 2003), p. 561-62.

15. Voir par exemple kim Nak Nyeon 金洛年, Nihon-teikokushugi-ka no chōsen keizai 日本帝国主義 下の朝鮮経済 (L'économie coréenne sous l'impérialisme japonais), Tōkyō daigaku shuppan, 2002, 245 p. ; et sa recension par Iwamoto Takunari 岩本卓也 dans Ajia kenkyū アジア研究 (Études asiatiques), vol. 50, no. 4 (oct. 2004), p. 98-102.

16. Ce dont on peut douter à la lecture d'un travail sud-coréen (économétrique) de cette mouvance prenant radicalement le contre-pied de l'école "nationaliste " en affirmant en introduction que l'État colonial était « efficace, sinon démocratique ». cha Myung Soo et Kim Nak Nyeon, “Korea's First Industrial Revolution, 1911-40", Explorations in Economic History, no. 49, 2012, p. 60-74, p. 60.

17. Signe de la banalisation de cette focalisation, cet article publié récemment dont le titre est dépourvu de délimitation chronologique bien qu'il traite des années 1920 et 1930: Takeuchi Yūsuke 竹内祐介, «Kokubutsu-jukyū wo meguru nihon-teikoku-nai-bungyō no saihensei to 
shokuminchi chōsen» 殸物需給をめぐる日本帝国内分業の再編成と植民地朝鮮（La restructuration du marché des grains au sein de l'empire japonais et la Corée coloniale), Shakai keizai-shigaku 社会経済史学 (Études en histoire socio-économique), vol.74, no. 5 (janv. 2009), p. 447-467.

18. Concernant la colonisation agricole, voir Alexandre Roy, «L'implantation des colons agricoles en Corée entre 1905 et 1919 : l'histoire d'un échec », dans Jean-Michel Butel et Makiko Ueda (dir.), Japon Pluriel, $n^{\circ} 9$, Picquier, à paraître.

19. Sur Shibusawa dans cette affaire, voir Claude Hamon, Shibusawa Eiichi (1840-1931). Bâtisseur du capitalisme japonais, Maisonneuve \& Larose, 2007, 379 p., p. 264-266.

20. Takahashi Yasutaka 高橋泰隆, «Shokuminchi tetsudō to kaiun» 植民地鉄道と海運 (Les chemins de fer et le transport maritime des colonies), dans ōe Shinobu (dir.), Kindai Nihon to shokuminchi, op. cit., p. 271.

21. Propos de Shibusawa en 1908, cité dans Claude Hamon, Shibusawa..., op. cit., p. 267.

22. Takahashi Yasutaka, «Shokuminchi tetsudō to kaiun », op. cit., p. 272.

23. Ibid., p. 269-271.

24. Claude Hamon, Shibusawa..., op. cit., p. 272-73.

25. Ce paragraphe regroupe des informations tirées de Peter Duus, The Abacus and the Sword. The Japanese Penetration of Korea, Berkeley, University of California Press, 1995, XIV + 480 p., p. 155-157. 26. Chōsen sōtoku-fu tetsudō-kyoku 朝鮮総督府鉄道局 (Bureau des Chemins de fer, Gouvernement général de Corée), Chōsen tetsudō-shi 朝鮮鉄道史 (Histoire des chemins de fer de Corée), Keijō, 1929, 766 p, p. 302.

27. chōsen sōtoku-fu, Chōsen sōtoku-fu shisei nenpō 朝鮮総督府施政年報 (Annuaire statistique du Gouvernement général de Corée), années 1918-1920, vol. 8, p. 237.

28. Yoshihisa T. Matsusaka, The making of Japanese Manchuria, 1904-1932, Harvard University Asia Center, 2001, 544 p., pp. 41-49.

29. Bank of Chosen, Economic History of Chosen, Keijō, 1920, 266 p., p. 102.

30. Hirai Kōichi 平井廣一, «Nihon shokuminchi-ka ni okeru chōsen tetsudō zaisei no tenkai katei »日本植民地下における朝鮮鉄道財政の展開過程 (L'évolution financière des chemins de fer coréens sous la colonisation japonaise), Keizai-gaku kenkyū 経済学 研究, vol.34, no.4, mars 1985, Hokkaidō daigaku, p. 12-32, p. 16.

31. Constat que l'on retrouve jusque dans le Recueil sur le chemin de fer coréen publié par les autorités coloniales en 1930 (Chōsen sōtokufu tetsudō-kyoku, Chōsen tetsudō ronsan 朝鮮鉄道論 纂, Keijō, 1930, 536 p., p. 168).

32. Chōsen ginkō 朝鮮銀行 (Banque coloniale de Corée), Senman keizai jūnen-shi 鮮満経済十年史 (Histoire économique de Corée et Mandchourie sur les dix dernières années), Keijō, 1919, 461 p., p. 59.

33. Hirai Kōichi, « Nihon shokuminchi-ka... », op. cit., p. 17.

34. Sur une demande moyenne de 930000 tonnes entre 1918 et 1920. Chōsen sōtoku-fu tetsudōkyoku, Chōsen tetsudō ronsan, op. cit., p. 201.

35. Bank of Chosen (Banque coloniale de Corée), Economic History of Chosen, op. cit., p. 180-181.

36. Les chiffres suivants sont tirés de l'annuaire statistique du gouvernement général (années 1918-1919).

37. La terminologie coloniale distinguait les exports et imports commerciaux coloniaux (ishutsu 移出 et inyū 移入) des mouvements commerciaux extérieurs (yushutsu 輸出 et yunyu $\bar{u}$ 輸入). L'abolition de cette taxe a été décidée dans le cadre du développement des exportations de riz vers le Japon, voir notre dernière partie.

38. Government-General of Chosen, Annual Report on Reforms and Progress in Chosen, Keijō, vol. de l'année 1922, 294 p., p. 59-60.

39. Pour les chiffres précis, voir ibid., p. 37-39. 
40. 7,7 millions de yens en 1916 ; 9,9 en 1917. Bank of Chōsen, Economic Outlines of Chosen and Manchuria, Keijō, 1918, 44 p., p. 11.

41. Les chiffres du commerce coréen sont disponibles dans Yamamoto Yūzō, Shokuminchi keizai-shi kenkyū 植民地経済研究 (Études en histoire économique des colonies), Nagoya daigaku shuppankai, 1992, 313 p., p. 228.

42. Pour tout ce qui a trait à la Banque $\mathrm{n}^{\circ} 1$ en Corée, voir Claude Hamon, Shibusawa..., op. cit., p. 251-55, 258-62 et 276.

43. Ibid., p. 254.

44. Bank of Chosen, Economic History of Chosen, op. cit., p. 48.

45. Ibid., p. 50.

46. Peter Duus, The Abacus and the Sword, op. cit., p. 167.

47. Claude Hamon, Shibusawa..., op. cit., p. 262.

48. Bank of Chosen, Economic History of Chosen, op. cit., p. 55.

49. Ibid., p. 53. Voir aussi Government-general of Chosen, Annual Report on Reforms..., op.cit., p. 63.

50. Bank of Chosen, Economic History of Chosen, op. cit., p. 49, 54-55.

51. Bank of Chosen, Economic Outlines of Chosen and Manchuria, op. cit., p. 31-32.

52. Chōsen étant le nom officiel donné par le Japon à la Corée coloniale (il s'agissait de nier la «Corée » en tant qu'État national - kankoku 韓国 - pour la limiter à son espace géographique, ce à quoi renvoie chōsen, comme dans chōsen hantō 朝鮮半島, "la péninsule coréenne »), nous traduisons donc Chōsen ginkō par «Banque coloniale de Corée ». Précisons que les documents japonais d'époque de langue française ou anglaise utilisaient l'expression «Chosen " (comme dans nos trois précédentes références).

53. Bank of Chosen, Economic History of Chosen, op. cit., p. 189.

54. Ibid., p. 70.

55. Ibid., p. 183-184.

56. Kim Nak Nyeon (dir.), op. cit., p. 386.

57. $\operatorname{CSTN}$, chaque année.

58. «À la Bourse, il est arrivé de s'arracher les actions d'entreprises arborant le mot "Corée" sans même s'enquérir de leurs activités ", Tōkyō keizai zasshi 東京経済䧱誌 (L’Économiste de Tōkyō), no. 302 (13 déc. 1919), cité dans Kaneko Fumio, « Dai-ichi taisen-go no tai-shokuminchi tōshi » 第 一大戦後の対植民地投資 (Les investissements en colonies dans l'après-Première guerre mondiale), Shakai-keizai shigaku, vol. 51, no. 6 (mars 1986), reprod. dans okabe Makio 岡部牧夫 (éd.), Teikokushugi to shokuminchi 帝国主義と植民地 (Impérialisme et colonies), Tōkyō-dō shuppan 東京堂出版, 2001, 423 p., p. 138.

59. Les trois quarts des crédits accordés par les banques de Corée entre 1910 et 1920 le furent pour le secteur commercial. Kimura Mitsuhiko, "Financial Aspects of Korea's Economic Growth under Japanese Rule", Modern Asian Studies, vol. 20, no 4, 1986, pp. 793-820, p. 808.

60. Pour la population en 1920 nous retenons un chiffre de 18 millions d'habitants, moyenne de l'estimation de Cha Myung Soo (18,6 millions d'hab.) et du chiffre des statistiques officielles (17,3 millions d'hab.).

61. Un examen micro-analytique de ces entreprises permettrait de vérifier ou infirmer ce point.

62. Toutes les données de ce paragraphe sont à nouveau calculées à partir des chiffres disponibles dans les annuaires statistiques du gouvernement général (CSTN) de chaque année (1911-1919).

63. Government-General of Chosen, Annual Report on Reforms..., op. cit., notamment p. 118-123.

64. «Chōsen no minkan-kōjō » 朝鮮の民間工場 (Les usines privées de Corée), Keijō nippō 京城日 報, 9 février 1921 . 
65. «Chōsen no kōgyō » 朝鮮の工業 (L’industrie coréenne), Fukuoka nichinichi shinbun 福岡日日 新聞, 10 mars 1918.

66. «La crise du riz au Japon », Bulletin économique de l'Indochine (BEI dorénavant), vol. 15, nº 99, nov.-déc. 1912, p. 907-908.

67. Idem.

68. Hayami Yujirō, V. W. Ruttan, “Korean Rice, Taiwan Rice, and Japanese Agricultural Stagnation: An Economic Consequence of Colonialism", The Quarterly Journal of Economics, vol. 84, no 4, Nov. 1970, p. 562-589, p. 566.

69. Le koku 石 est une unité de volume équivalant à 180,39 litres généralement admis au Japon comme pesant $140 \mathrm{~kg}$. Toutefois, selon un expert indochinois, un koku de riz décortiqué pesait environ $108 \mathrm{~kg}$ et un koku de riz non décortiqué $130 \mathrm{~kg}$. Voir «L'importation et l'exportation du riz au Japon en 1912 », BEI, vol. 16, n 101, mars-avril 1913, p. 255. Nous préférons donc éviter la conversion en kilogramme pour garder le principe voulant qu'un koku de riz corresponde à la quantité annuelle nécessaire à un adulte (un demi-litre de riz - non cuit - par jour).

70. «Importations et exportations de riz au Japon en 1908, 1909 et 1910 », BEI, nº 91, juilletaoût 1911, vol. 14, p. 91.

71. Ōkura-shō sozei-kyoku 大蔵省租税局 (Bureau des Impôts du Ministère des Finances), Gaikoku bōeki gairan 外国貿易外覧 (Aperçu général du commerce extérieur), année 1911, p. 436.

72. Voir les articles suivants parus dans le BEI: «La question du riz au Japon», vol. 13, $\mathrm{n}^{\circ} 87$, nov.-déc. 1910 ; « La récolte du riz au Japon », vol. 14, n 88, janv.-fév. 1911, p. 96 ; « Importations et exportations de riz au Japon en 1908, 1909 et 1910 », op. cit., p. 758 ; « La crise du riz au Japon », op.cit., p. 907-910 ; «L'importation et l'exportation du riz au Japon en 1912 », vol. 16, nº 101, mars-avril 1913, p. 254-255; « Le riz et la question du riz au Japon », vol. 16, nº 102, mai-juin 1913, p. 271-278 ; « Le riz au Japon », vol. 17, n 108, juin-juillet 1914, p. 302-303.

73. « La crise du riz au Japon », BEI, op. cit., p. 907-908.

74. Nōshōmu-shō nōmu-kyoku 農商務省農務局 (Bureau de l'agriculture, ministère de l'Agriculture et du Commerce), Kome no tōkei tekiyō 米ノ統計摘要 (Abrégé pratique des statistiques du Riz), vol. 2, 1920, 10 p. Pour un compte-rendu plus textuel voir Liu Zhaoyan 劉照 彦, Nihon teikokushugi-ka no taiwan 日本帝国主義下の台湾 (Taiwan sous l'impérialisme japonais), Tōkyō daigaku shuppan, 1975, 502 p., p. 77.

75. Kazama Hideto 風間秀人, 《Shokuminchi jinushi to nōgyō» 植民地地主制と農業 (Les propriétaires terrains et l'agriculture dans les colonies), in Asada Kyōji 浅田喬二 (dir.), Kindai Nihon no kidō 近代日本の軌道 (La trajectoire du Japon moderne), Yamakawa shuppan 山川出版, 1994, vol. 10, 296 p., p. 108-130, p. 120.

76. Liu Zhaoyan, Nihon teikokushugi-ka no taiwan, op. cit., p. 87.

77. « Le riz et la question du riz au Japon », BEI, op. cit., p. 272.

78. Plus d'un million de koku de riz indochinois importé en 1913, mais moitié moins en 1914, puis moins de cent mille.

79. Chōsen sotokufu nōrinkyoku 朝鮮総督府農林局 (Bureau de l'Agriculture, Bois et Forêts du Gouvernement général de Corée), Chōsen no nōgyō 朝鮮の農業 (L'agriculture coréenne), vol. de l'année 1941, 323 p., p. 189-191.

80. Government-General of Chosen, Annual Report on Reforms..., op. cit., p. 103.

81. Entre les périodes 1912-1916 et 1917-1921, la part de la croissance de la production rizicole coréenne partie en exportations était de près des deux tiers (63\%), mais de moins d'un cinquième (17 \%) pour la croissance de la production céréalière. Les imports coréens de céréales triplèrent en volume alors que les imports de riz chutèrent d'autant, signe que la consommation coréenne se rabattait sur les denrées les moins chères, les moins demandées par la métropole. Nous suivons ici les chiffres disponibles dans Chōsen sotokufu nōrinkyoku, Chōsen no nōgyō..., op. cit., p. 189-191. 
82. Yahata八幡. La transcription "Yawata », que l'on trouve souvent, est une lecture erronée dans le cas de ce toponyme.

83. Kitakyūshū shishi 北九州市史 (Histoire de la ville de Kitakyūshū), «Sangyō keizai » 産業経済I (Économie et industrie I), Kitakyūshū-shi, 1991, 1318 p., p. 332.

84. Voir «Chōsen no tekkō» 朝鮮の鉄鉱 (Le minerai de fer coréen), ōsaka mainichi shinbun, 3 février 1917. Asō Tarō 麻生太郎, premier ministre japonais de septembre 2008 à septembre 2009 est le descendant de la famille Asō en question.

85. Via la Banque Industrielle du Japon (nihon kōgyō ginkō 日本興行銀行), les intérêts étant payés par l'exportation de minerai vers le Japon (70 000 tonnes minimum par an à partir de 1904, sur quinze ans; modifié à partir de 1913 en 10,5 millions de tonnes et 8 millions de tonnes de fonte sur quarante ans). «Seitetsujo shisatsu » 製鉄所視察 (Une visite des aciéries [de Yahata]), Chūgai shōgyō shinpō 中外商業新報 (Nouvelles commerciales d'ici et d'ailleurs), série de 10 articles, 26 juillet - 4 août 1915 .

86. Le minerai était extrait d'un gisement découvert par un Japonais qui en avait obtenu la concession et vendait toute sa production aux aciéries de Yahata. Nagura Bunji 奈倉文二, Nihon tekkō-gyō to nanyō tekkō shigen 日本鉄鋼業と「南洋」鉄鋼資源 (L'industrie sidérurgique japonaise et les ressources ferrifères des Mers du Sud), Tōkyō, Kokusai rengō daigaku (Université des Nations Unies), 1981 (ningen to shakai no puroguramu kenkyū hōkoku-sho 33 - Projet sur l'expérience japonaise en matière de transfert, transformation et développement de la technologie : rapport de recherches $\left.n^{\circ} 33\right), 54 \mathrm{p}$.

87. “Kōzai kyōkyū-mondai, tetsu no kikin» 鋼材供給問題 鉄の饑餀 (Le problème de l'approvisionnement en acier : la famine du fer), Jiji shinpō 時事新報, série de trois articles, 17 19 juillet 1916.

88. Une moyenne de 99 yens la tonne à l'importation sur 1912-15, 174 yens en 1916, 281 yens en 1917. Shōkō-shō kōzan-kyoku, Seitetsu-gyō sankō shiryō, op. cit.

89. Sur la question de l'embargo, voir Jeffrey J. safford, "Experiment in Containment: The United States Steel Embargo and Japan, 1917-1918”, The Pacific Historical Review, vol. 39, no. 4, Nov. 1970, p. 439-451.

90. 377 yens la tonne en 1918. shōkō-shō kōzan-kyoku, Seitetsu-gyō sankō shiryō, op. cit.

91. «Seitetsu-netsu no bokkō » 製鉄熱の勃興 (Le boum de la fièvre sidérurgique), Ōsaka mainichi shinbun, 24 août 1918. On évoqua aussi le sujet dans la presse indochinoise : « Les fluctuations du marché de l'acier au Japon en 1917 », BEI, vol. 21, nº 131, juillet-août 1918, p. 725.

92. Mitsubishi shōji kabushiki-gaisha 三菱商事株式会社 (Maison commerciale Mitsubishi, $\mathrm{S}^{\mathrm{té}^{\mathrm{e}}}$ par actions), Mitsubishi shōjisha-shi 三菱商事社史 (L'histoire de la Maison commerciale Mitsubishi), tome 1, 1986, p. 103 ; et ibid., volume « Shiryō » 資料 (Documents), 1987, p. 186 et p. 188.

93. Ibid., p. 103 et 147.

94. Government-General of Chosen, Annual Report on Reforms..., op. cit., p. 120.

95. «Seitetsu jigyō no botsuraku » 製鉄事業の没落 (Le naufrage du secteur sidérurgique), Ōsaka asahi shinbun, 23 novembre 1919.

96. «Il ne fait aucun doute qu'avec la fièvre entrepreneuriale, [la spéculation] a épuisé les affaires en métropole et a finalement atteint les colonies ", Tōkyō keizai zasshi, no. 302 (13 déc. 1919), cité dans Kaneko Fumio, « Dai-ichi-taisen-go... », op. cit., p. 138.

97. C'est ce que révèlent les «Propos recueillis dans les bistrots» (shumakusō 酒幕談叢; les "shumaku», chumak en coréen, étant les lieux populaires de boissons en Corée) établis secrètement par la gendarmerie japonaise (kempeitai 憲兵隊). Matsuda Toshihiko, Governance and policing of Colonial Korea: 1904-1919, Nichibunken 日文研, 2011, 256 p., p. 132-149.

98. 《[Senman] kakuchi no bukka»[鮮満]各地の物価 (Le coût de la vie [en Corée et Mandchourie]), Keijō nippō, 28 février 1919 (dernier article d'une série de cinq parue à partir du 15 février). 
99. Voir l'article de Pierre François Souyri dans le présent numéro de Cipango.

100. Titre de son article publié dans le Tōyō keizai shinpō 東洋経済新報 (Les Nouvelles économiques d'Orient), 30 juillet 1921, cité dans Narita Ryūichi 成田龍一, Taishō demokurashî 大正 デモクラシー (La démocratie Taishō), Iwanami shoten, 2008, 258 p., p. 162.

\section{RÉSUMÉS}

Pour cette première étude en français de l'économie coloniale japonaise, nous avons choisi de l'analyser à partir du cas de la Corée depuis l'aménagement des premiers rails japonais sur place en 1900 jusqu'à l'éclatement de la crise globale du joug colonial au lendemain de la Première Guerre mondiale (1919). La colonisation économique de la Corée promettait un développement important sur une terre étendue, peuplée et proche de la métropole. Elle a pourtant rapidement buté sur un certain nombre d'obstacles. Les infrastructures coloniales fondamentales, les finances et le chemin de fer, ont souffert tantôt d'un manque d'investissements, tantôt de la politique mandchourienne promue par Terauchi Masatake. La production industrielle et manufacturière resta marginale, malgré son développement relativement précoce et important : stimulé par une fièvre boursière à partir de 1916, le secteur s'effondra avec l'éclatement de la bulle en 1919. Commercialement, la colonie fut déficitaire en permanence et de manière exponentielle. La métropole n'y voyait qu'une source d'approvisionnements en riz et minerai de fer, alors même que la production coréenne ne pouvait concurrencer l'offre environnante (Indochine et Taiwan pour le riz, Chine pour le fer)... du fait du manque d'investissements. Cette faiblesse de la métropole en Corée rendit la domination japonaise particulièrement insupportable : l'effondrement de la bulle métropolitaine suivant la fin de la guerre mondiale fut aussi celui de tout espoir socio-économique en Corée. L'inflation galopante et l'absence de véritable politique de développement favorisèrent et précipitèrent l'avènement du Mouvement du $1^{\mathrm{er}}$ Mars 1919.

\section{INDEX}

Index géographique : Corée

Mots-clés : colonie, industrie, politique coloniale

Keywords : Korea, History, Colony, Economy, Industry, Colonial Policy, Meiji Period, Taishō

Period

Thèmes : histoire, économie

Index chronologique : Meiji (1868-1912), Taishō (1912-1923)

\section{AUTEUR}

\section{ALEXANDRE ROY}

Centre d'études japonaises, Inalco

Université de Toulouse-Le-Mirail 